$\underline{\text { Preprint typeset in JHEP style - HYPER VERSION }}$

DAMTP-2007-42

TIFR/TH/07-08

\title{
$Z$ boson decay to photon plus Kaluza-Klein graviton in large extra dimensions
}

\author{
Benjamin C. Allanach and Jordan P. Skittrall \\ Department of Applied Mathematics and Theoretical Physics, \\ Centre for Mathematical Sciences, Univeristy of Cambridge, \\ Wilberforce Road, Cambridge, CB3 0WA, United Kingdom \\ E-mail: B.C.Allanach@damtp.cam.ac.uk, J.P.Skittrall@damtp.cam.ac.uk \\ K. Sridhar \\ Department of Theoretical Physics, \\ Tata Institute of Fundamental Research, \\ Homi Bhabha Road, Bombay 400005, India \\ E-mail: sridhar@theory.tifr.res.in
}

\begin{abstract}
In the large extra dimensional ADD scenario, Z bosons undergo a one-loop decay into a photon and Kaluza-Klein towers of gravitons/gravi-scalars. We calculate such a decay width, extending previous arguments about the general form of the fourdimensional on-shell amplitude. The amplitudes calculated are relevant to processes in other extra dimensional models where the Standard Model fields are confined to a 4-brane.
\end{abstract}

Keywords: Large Extra Dimensions, Beyond Standard Model. 


\section{Contents}

1. Introduction 2

1.1 General notation

2. Fermion loop diagrams 7

3. W loop diagrams 11

4. Counterterm diagrams 15

5. General forms of the amplitudes 19

5.1 Decay into spin-0 excitation and photon 19

5.2 Decay into spin-2 excitation and photon 20

6. Transversality conditions 21

6.1 Electromagnetic transversality: spin-2 case 21

6.1 .1 Fermion loop diagrams 21

6.1.2 W loop diagrams

6.1.3 Counterterm diagrams 23

6.2 Gravitational transversality: spin-2 case 23

6.2.1 Fermion loop diagrams 23

6.2.2 W loop diagrams 23

6.2.3 Counterterm diagrams 24

6.3 Electromagnetic transversality: spin-0 case 24

6.3.1 Fermion loop diagrams 24

6.3.2 W loop diagrams 25

6.3.3 Counterterm diagrams 26

7. Calculation of the amplitude coefficients 26

7.1 Counterterm diagrams contribution 26

7.1.1 Spin-2 case 26

7.1.2 Spin-0 case 27

7.2 Loop diagram contributions to the spin-2 amplitude coefficients 27

7.2.1 Fermion loop diagrams 27

7.2.2 W loop diagrams 28

7.3 Overall contribution to the spin-2 amplitude coefficients 29

7.4 Loop diagram contributions to the spin-0 amplitude coefficients 31

7.4.1 Fermion loop diagrams 31

7.4.2 W loop diagrams 33

7.5 Overall contribution to the spin-0 amplitude coefficients 36 
8. Calculation of the decay width 37

8.1 Decays involving spin-2 Kaluza-Klein particles 37

8.2 Decays involving spin-0 Kaluza-Klein particles 39

8.3 Overall decay width to photon and Kaluza-Klein graviton excitation 40

9. Conclusions 43

A. Dimensional regularization integral identities 44

\section{Introduction}

Nieves and Pal [1] have considered the decay of a $\mathrm{Z}$ boson to a photon and a standard four-dimensional graviton. In the ADD scenario [2] $]^{1}$, the graviton may be viewed from a four-dimensional perspective as gaining a "tower" of massive Kaluza-Klein (KK) excitations [6, 7] (this tower takes a relatively simple form if we assume that the extra dimensions are toroidally compactified with the copies of $S^{1}$ having a common radius $R / 2 \pi$, with $R$ the common circumference). Although the decays of a $\mathrm{Z}$ boson involving real production of a KK graviton excitation with a photon will be suppressed by a gravitational coupling, the existence of a "tower" of particles to which the $\mathrm{Z}$ boson can decay may counteract this suppression. Because the KK excitations only couple with gravitational strength to Standard Model particles, they will almost certainly pass through a detector (their detection is a next-to-leading-order process in the gravitational coupling). The ADD scenario therefore predicts that we should see an increase in the decay width of the $\mathrm{Z}$ boson to a photon and missing energy relative to the Standard Model prediction [8, 9, 10, 11].

In this paper, we calculate, to leading order, each of the decay widths of the $\mathrm{Z}$ boson to a photon and a KK graviton excitation. There are two relevant towers of KK excitations: a spin-2 tower and a spin-0 tower. We combine the calculated widths to obtain an overall decay width for the decay of a $\mathrm{Z}$ boson to a photon and some KK graviton excitation. This calculated width will allow the determination of bounds on the size of large extra dimensions when combined with experimental data on $\mathrm{Z}$ decay [12]. (The current upper limit on the branching ratio for $Z \rightarrow \gamma+X$, with $X$ some beyond-Standard Model invisible particle or particles, is $O\left(10^{-6}\right)$ [13]. With a "Giga-Z" collider setup, this could potentially be reduced to around $O\left(10^{-9}\right)$.) The amplitudes calculated are also relevant to processes in other extra dimensional models where the Standard Model fields are confined to a 4-brane (e.g. the Randall-Sundrum 1 (RS1) model [14]).

Current experimental limits on the size of ADD extra dimensions from processes other than $\mathrm{Z}$ boson decay come from inverse square law experiments [15, 16], from consideration of the channel $e^{+}+e^{-} \rightarrow \gamma+$ graviton at the LEP experiments [17, and from consideration

\footnotetext{
${ }^{1}$ The idea of large extra dimensions was also considered 3, 4, 贯 prior to the work of Arkani-Hamed, Dimopoulos and Dvali.
} 
of the channel $p+\bar{p} \rightarrow$ jet+graviton at the Tevatron experiments 18, 19. In the near future, the most likely improvement in these experimental bounds should come with the publication of results from the D $\varnothing$ experiment using Run II of the Tevatron, and combination of those results with the already published CDF Run II bounds [18]. Further into the future, we can expect investigation of large extra dimensions at the Large Hadron Collider.

For the decays considered in this paper, the leading order process is a one-loop process, because the tree-level vertex for $\mathrm{Z}$ boson decay into a photon and a graviton is absent. This means that there is a higher order prefactor in coupling constants of the decay width when this decay mode is compared to other, tree-level, graviton production modes considered previously [6, 7, 20]. However, there is a large amount of experimental data for $\mathrm{Z}$ boson decay, which makes reasonable a comparison of bounds set by this novel production process with bounds set by other processes.

The tree-level vertex is absent because it is derived by considering a perturbative expansion of the metric about flat space, so that the Z- $\gamma$-graviton term comes from a perturbation of the Z- $\gamma$ Lagrangian term, which is zero. (In essence, each vertex involving a KK graviton excitation is derived by "hanging" a graviton off a propagator or an existing vertex [21, 22].) Because we are working with bare parameters, we are in effect working with a basis for the electroweak sector in which there is $\mathrm{Z}-\gamma$ mixing at one-loop level, but this will only enter the Lagrangian via counterterm corrections. It is clear that the amplitude calculated will not contain terms associated with Z- $\gamma$-graviton tree-level mixing, since we could calculate the amplitude using renormalized parameters instead of counterterms, whereby we should have explicitly no Z- $\gamma$-graviton mixing.

At leading order, the decay is either into a spin-2 KK excitation or into a spin-0 KK excitation (the spin-1 KK excitation does not couple directly to matter [0]). The spin2 case is almost identical to the case of $\mathrm{Z}$ boson decay to photon plus graviton without extra dimensions, which has been considered by Nieves and Pal [1]. We repeat in this paper some of the detail, for sake of completeness. The methodology of the spin- 0 case is strongly motivated by that of the spin-2 case.

In many tree-level decays involving particles with small masses, the contribution to the decay width of channels involving a spin-0 KK graviton can be ignored, as the vertices involving the spin-0 KK graviton contain the masses of the other particles (and in some cases also contain momentum terms that are zero on-shell). However, the possibility of massive particles in the loop means that there is a non-negligible contribution to the amplitude in the one-loop calculation from the spin-0 decay channel.

For the decays into a spin-2 KK graviton and for the decays into a spin-0 KK graviton, three types of diagram must be considered. The first two types of diagram are those with a fermion in the loop and those with a $\mathrm{W}$ boson in the loop. (In principle, the second type of diagram also includes diagrams with Goldstone bosons and Fadeev-Popov ghosts in the loop, but we adopt the unitary gauge throughout.) A third type of diagram is required if we are to work using bare parameters, namely the diagrams containing a counterterm. It will turn out that the counterterm diagrams evaluate to zero, which is why we work with bare parameters (and not renormalized parameters). The result that these diagrams evaluate to zero supports the conclusion in [1] that such diagrams need not be considered 
in the near-flat Standard Model scenario, and supports what appears to be a "miraculous cancellation" of divergent terms in the spin-0 KK graviton calculation in this paper.

We also make use of the argument of [1] that, in the spin-2 KK graviton case, conservation of the electromagnetic current and of the energy-momentum tensor implies a particular general form taken by the amplitude, which simplifies the calculation. We derive an analogous argument for the spin-0 KK graviton case.

It is possible to estimate the form that the decay width will take prior to a full calculation. For the decay of a $\mathrm{Z}$ boson into a photon and a graviton in $3+1$-dimensional space, Nieves and Pal [1] give the estimate

$$
\Gamma \sim \alpha^{2} G M_{Z}^{3}
$$

by dimensional analysis. They derive this estimate by noting that the graviton coupling introduces into the amplitude a factor of $\kappa=\sqrt{8 \pi G}$, where $G$ is Newton's constant in four dimensions; they also note that both the $\mathrm{Z}$ coupling and the photon coupling introduce into the amplitude a factor of $\sqrt{\alpha}$, where $\alpha$ is the fine-structure constant. (The estimate is then obtained by noting that the $\mathrm{Z}$ mass, $M_{Z}$, is the only dimensionful parameter remaining in the calculation.)

For the calculation in the ADD scenario, the factor $\alpha^{2} G$ remains in the width (and $G$ is still Newton's constant in four dimensions). However, we sum over the Kaluza-Klein excitations of the graviton by using an integral approximation over a "density" $\rho$ [7], and this density contains a factor $R^{n}$, where $R / 2 \pi$ is the radius of the extra dimensions and $n$ is the number of extra dimensions. The $\mathrm{Z}$ mass remains the only other dimensionful parameter (since we have summed over all KK graviton masses). We therefore obtain the estimate

$$
\Gamma \sim \alpha^{2} G R^{n} M_{Z}^{3+n} .
$$

The detailed phenomenology of our more precisely calculated results is covered in reference [12].

The model of toroidal compactification of the extra dimensions with a common compactification radius $R / 2 \pi$ is something of a toy model, not least because it does not suggest a mechanism for confining the Standard Model fields to a 4-brane, as is required for the ADD scenario. However, the model still deserves phenomenological investigation, firstly because the calculations involved may be useful for understanding models where the topology of the compactified dimensions is more complicated, and secondly because the toy model still gives some indication of the likely consistency of the ADD scenario with experimental observations.

This paper is organised as follows. In Sections 2, 3 and 4, we state the Feynman rules required and give the diagrams corresponding to a fermion loop, a $\mathrm{W}$ boson loop and a counterterm, respectively. In Section 5 we present arguments giving the general form of the amplitude in each of the spin-2 KK graviton production and the spin-0 KK graviton production cases. Consideration of the spin-2 case, identically to that derived by Nieves and Pal [1], shows that the amplitude can be expressed in terms of one CP-even and two $\mathrm{CP}$-odd coefficients. Consideration of the spin-0 case shows that the amplitude can be 
expressed in terms of one CP-even and one CP-odd coefficient. In Section 6, we demonstrate that the sets of diagrams previously presented satisfy the relevant electromagnetic and gravitational transversality conditions, so that it is possible to use the general form arguments. We then proceed in Section 7 to calculate the coefficients required for an expression of the amplitudes. In Section 8, we use the expressions for the amplitudes to calculate decay widths for the individual KK excitation modes, and then sum over these modes (approximating the sum by an integral [7]) to obtain an overall decay width.

\subsection{General notation}

We take Greek indices to range over the four dimensions corresponding to the Standard Model brane, and Roman indices to range over the $n$ extra (bulk) dimensions.

We work with a metric tensor linearised so that

$$
g_{\lambda \rho}=\eta_{\lambda \rho}+2 \kappa\left(h_{\lambda \rho}+\eta_{\lambda \rho} \phi\right)
$$

(note that this is a hybrid of the notation of [1, 22], which do not have the extra dimensional dilaton term, and of [7], which differs by a factor of two). With this definition, the gravitational coupling $\kappa$ satisfies

$$
\kappa=\sqrt{8 \pi G},
$$

where $G$ is Newton's constant. The fields generated by the KK reduction may be expanded in Fourier modes and redefined in terms of massive fields $\tilde{h}_{\lambda \rho}^{\vec{n}}, \tilde{A}_{\mu i}^{\vec{n}}$ and $\tilde{\phi}_{i j}^{\vec{n}}$ (for the spin2 , spin-1 and spin-0 cases respectively) [7]. (The spin-1 field does not couple directly to matter and is therefore neglected from now on as a higher order contribution.) $\vec{n}$ is a vector giving the excitation level in each of the extra dimensions.

We note that at each mass level (i.e. for each distinct value of the excitation vector $\vec{n}$ ), there are one spin-2 KK graviton excitation $\tilde{h}_{\lambda \rho}^{\vec{n}}$ and $n-1$ spin-0 KK graviton excitations

$\tilde{\phi}_{i j}^{\vec{n}}$ to be considered. The $n-1$ factor comes from noting that the Standard Model particles couple only to the trace $\tilde{\phi}^{\vec{n}}$ of the spin-0 particles, and that one degree of freedom is lost owing to the linear dependence of the modes $\tilde{\phi}_{i j}^{\vec{n}}$. Equivalently, we may note that vertices involving the spin-0 particles always contain a $\delta_{i j}$ term, and each external spin-0 particle is accompanied by an extra-dimensional "polarisation tensor" $e_{i j}$, which satisfies the spin sum identity [0]

$$
\sum_{s=1}^{n(n-1) / 2} e_{i j}^{s} e_{i^{\prime} j^{\prime}}^{s *}=\frac{1}{2} P_{i i^{\prime}}^{\vec{n}} P_{j j^{\prime}}^{\vec{n}}+\frac{1}{2} P_{i j^{\prime}}^{\vec{n}} P_{j i^{\prime}}^{\vec{n}}
$$

with

$$
P_{i j}^{\vec{n}}=\delta_{i j}-\frac{n_{i} n_{j}}{\vec{n}^{2}}
$$

which satisfies

$$
P_{i j}^{\vec{n}} P_{j k}^{\vec{n}}=P_{i k}^{\vec{n}}, \quad P_{i i}^{\vec{n}}=n-1,
$$

so that when we calculate the modulus-squared of the amplitude, the terms carrying extra dimensional indices look like

$$
\delta_{i j} \delta_{i^{\prime} j^{\prime}}\left(\frac{1}{2} P_{i i^{\prime}}^{\vec{n}} P_{j j^{\prime}}^{\vec{n}}+\frac{1}{2} P_{i j^{\prime}}^{\vec{n}} P_{j i^{\prime}}^{\vec{n}}\right)
$$


and, using equation (1.7), this evaluates to $n-1$, as expected.

We define the polarisation tensors $\mathcal{E}^{\lambda \rho}(q), \varepsilon^{\nu}(k)$ and $\varepsilon_{\mathrm{Z}}^{\mu}(p)$ as corresponding to the spin-2 KK graviton excitation, the photon and the $\mathrm{Z}$ boson, respectively. The tensors satisfy

$$
\begin{aligned}
& \varepsilon^{\nu}(k) k_{\nu}=0, \\
& \varepsilon_{\mathrm{Z}}^{\mu}(p) p_{\mu}=0,
\end{aligned}
$$

and

$$
\mathcal{E}^{\lambda \rho}(q) q_{\lambda}=0, \quad \mathcal{E}^{\lambda \rho}(q) q_{\rho}=0
$$

The gravitational polarisation tensor is symmetric and traceless:

$$
\mathcal{E}^{\lambda \rho}(q)=\mathcal{E}^{\rho \lambda}(q), \quad \mathcal{E}^{\lambda \rho}(q) \eta_{\lambda \rho}=0
$$

The momenta satisfy the on-shell conditions

$$
\begin{aligned}
p^{2} & =M_{Z}^{2}, \\
k^{2} & =0 \\
q^{2} & =m_{\vec{n}}^{2}
\end{aligned}
$$

where $m_{\vec{n}}$ is the mass of the KK graviton excited to level $\vec{n}$, given for the toroidally compactified ADD scenario by []]

$$
m_{\vec{n}}^{2}=\frac{4 \pi^{2} \vec{n}^{2}}{R^{2}}
$$

Four-momentum conservation $(p=k+q)$ yields the on-shell identity

$$
2 k \cdot q=M_{Z}^{2}-m_{\vec{n}}^{2}
$$

We introduce notation for the off-shell amplitudes $F_{\lambda \rho \mu \nu}^{(h)}(q, k)$ (in the case of decay to a spin-2 KK excitation) and $F_{\mu \nu}^{(\phi)}(q, k)$ (in the case of decay to a spin-0 KK excitation), defined by

$$
\mathcal{M}^{(h)}(q, k)=\mathcal{E}^{\lambda \rho *}(q) \varepsilon^{\nu *}(k) \varepsilon_{Z}^{\mu}(p) F_{\lambda \rho \mu \nu}^{(h)}(q, k)
$$

and

$$
\mathcal{M}^{(\phi)}(q, k)=\varepsilon^{\nu *}(k) \varepsilon_{\mathrm{Z}}^{\mu}(p) F_{\mu \nu}^{(\phi)}(q, k),
$$

respectively. 


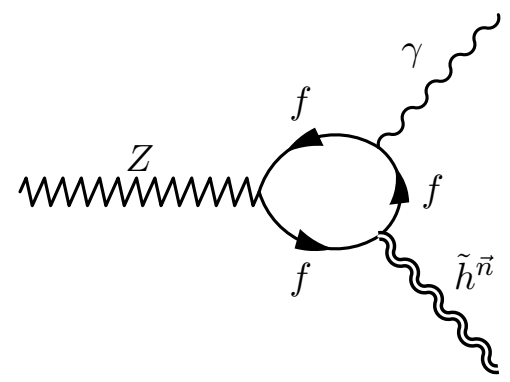

(a)

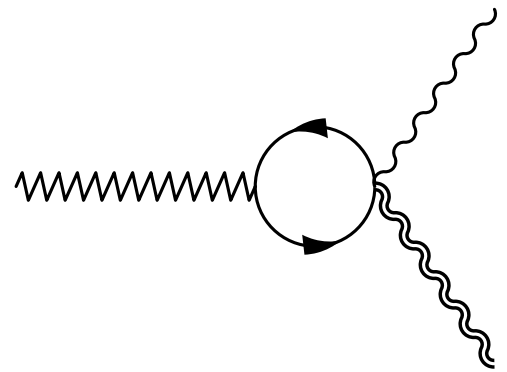

(c)

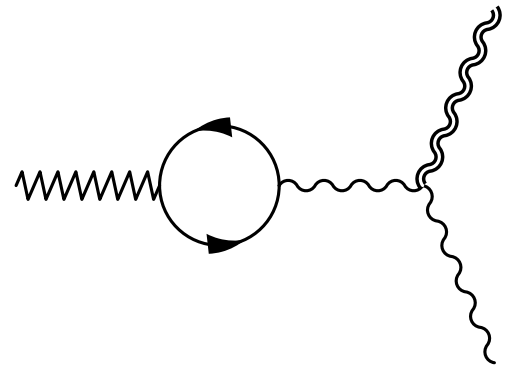

(e)

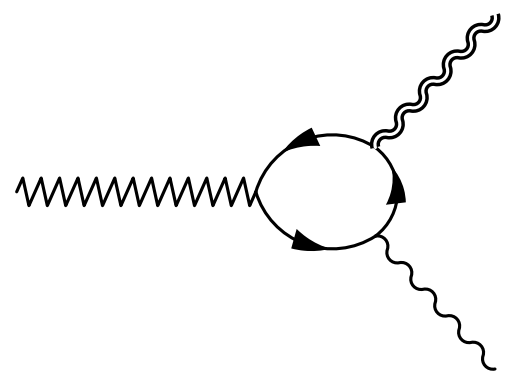

(b)

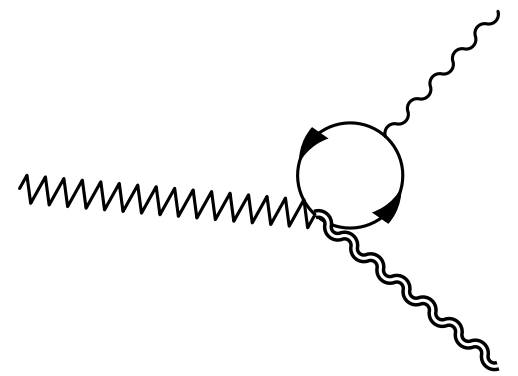

(d)

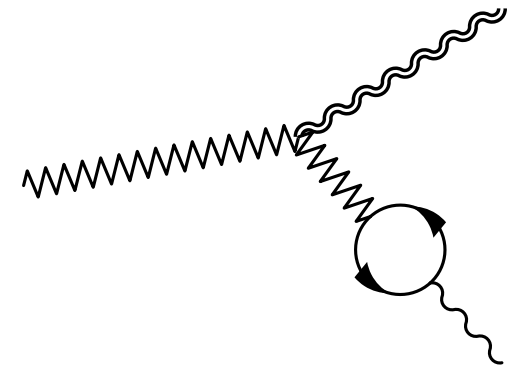

(f)

Figure 1: 1-loop diagrams for the process $Z \rightarrow \gamma+\tilde{h}^{\vec{n}}$ involving fermions in the loop. The spin-2 KK graviton excitations are represented by the double wavy lines.

\section{Fermion loop diagrams}

Figure 1 1 contains the diagrams for the $Z \rightarrow \gamma+\tilde{h}^{\vec{n}}$ process with a fermion in the loop, and figure 2 contains the diagrams for the $Z \rightarrow \gamma+\tilde{\phi}^{\vec{n}}$ process with a fermion in the loop.

The Feynman rules for the vertices occurring in the diagrams are given for reference in figure 3. Their derivation is given in [6, 7]. Some of the tensor contributions to the vertex factors are abbreviated for legibility; the abbreviations used are

$$
\begin{aligned}
C_{\lambda \rho \nu \sigma} \equiv & \eta_{\lambda \nu} \eta_{\rho \sigma}+\eta_{\lambda \sigma} \eta_{\rho \nu}-\eta_{\lambda \rho} \eta_{\nu \sigma} \\
c_{\lambda \rho \beta \nu}\left(k_{1}, k_{2}\right) \equiv & \left(k_{1} \cdot k_{2}\right) C_{\lambda \rho \beta \nu}+\eta_{\lambda \rho} k_{1 \nu} k_{2 \beta}- \\
& -\left[\eta_{\lambda \nu} k_{1 \rho} k_{2 \beta}+\eta_{\lambda \beta} k_{1 \nu} k_{2 \rho}-\eta_{\beta \nu} k_{1 \lambda} k_{2 \rho}+(\lambda \leftrightarrow \rho)\right], \\
c_{\lambda \rho \beta \nu}^{Z}\left(k_{1}, k_{2}\right) \equiv & c_{\lambda \rho \beta \nu}-M_{Z}^{2} C_{\lambda \rho \beta \nu}, \\
V_{\lambda \rho}\left(k_{1}, k_{2}\right) \equiv & \frac{1}{4}\left[\gamma_{\lambda}\left(k_{1}+k_{2}\right)_{\rho}+\gamma_{\rho}\left(k_{1}+k_{2}\right)_{\lambda}-2 \eta_{\lambda \rho}\left(k_{1}+\not k_{2}-2 m_{f}\right)\right] .
\end{aligned}
$$




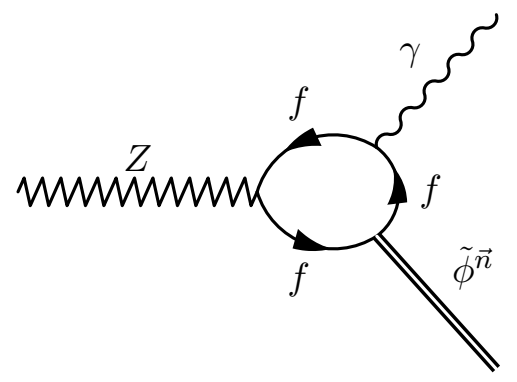

(a)

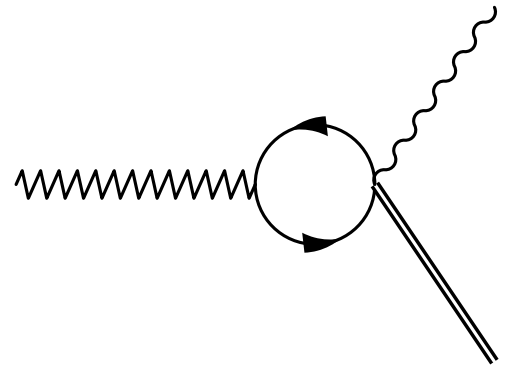

(c)

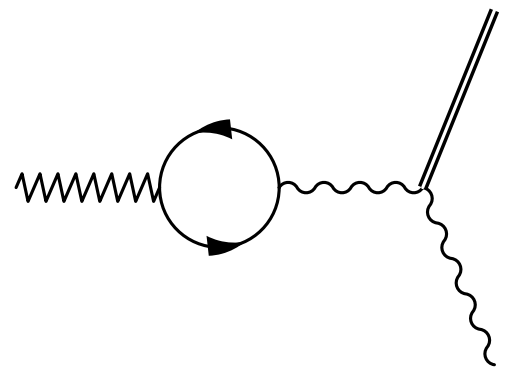

(e)

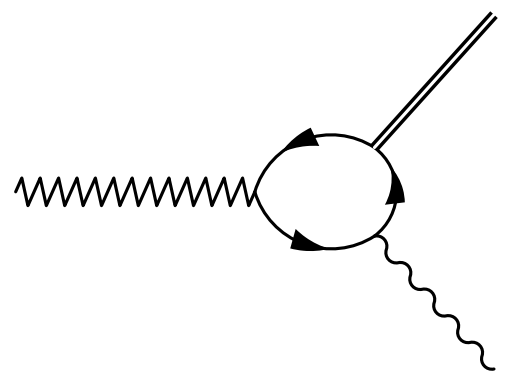

(b)

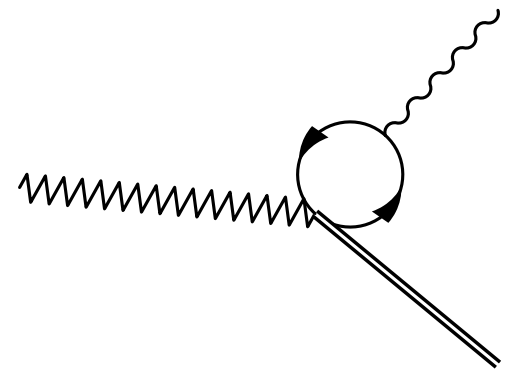

(d)

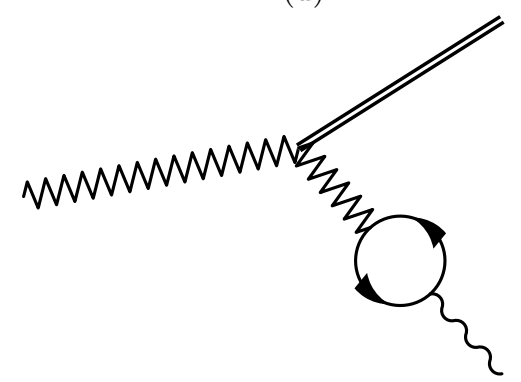

(f)

Figure 2: 1-loop diagrams for the process $Z \rightarrow \gamma+\tilde{\phi}^{\vec{n}}$ involving fermions in the loop. The spin-0 KK graviton excitations are represented by double straight lines.

We use $Q_{f}$ to denote the fermion charge, and have written

$$
\tilde{\gamma}_{\mu} \equiv \gamma_{\mu}\left(X_{f}+Y_{f} \gamma_{5}\right)
$$

$X_{f}$ and $Y_{f}$ are scalars. We have also defined (as in [7])

$$
\omega \equiv \sqrt{\frac{2}{3(n+2)}} .
$$

Writing $F_{\lambda \rho \mu \nu}^{(h f)}$ and $F_{\mu \nu}^{(\phi f)}$ to denote the contributions from the fermion loop diagrams to the off-shell amplitudes $F_{\lambda \rho \mu \nu}^{(h)}$ and $F_{\mu \nu}^{(\phi)}$, respectively, and absorbing constants by defining

$$
F_{\lambda \rho \mu \nu}^{(h f)} \equiv \frac{\kappa e Q_{f} g}{2 \cos \theta_{W}} T_{\lambda \rho \mu \nu}^{(h f)}
$$




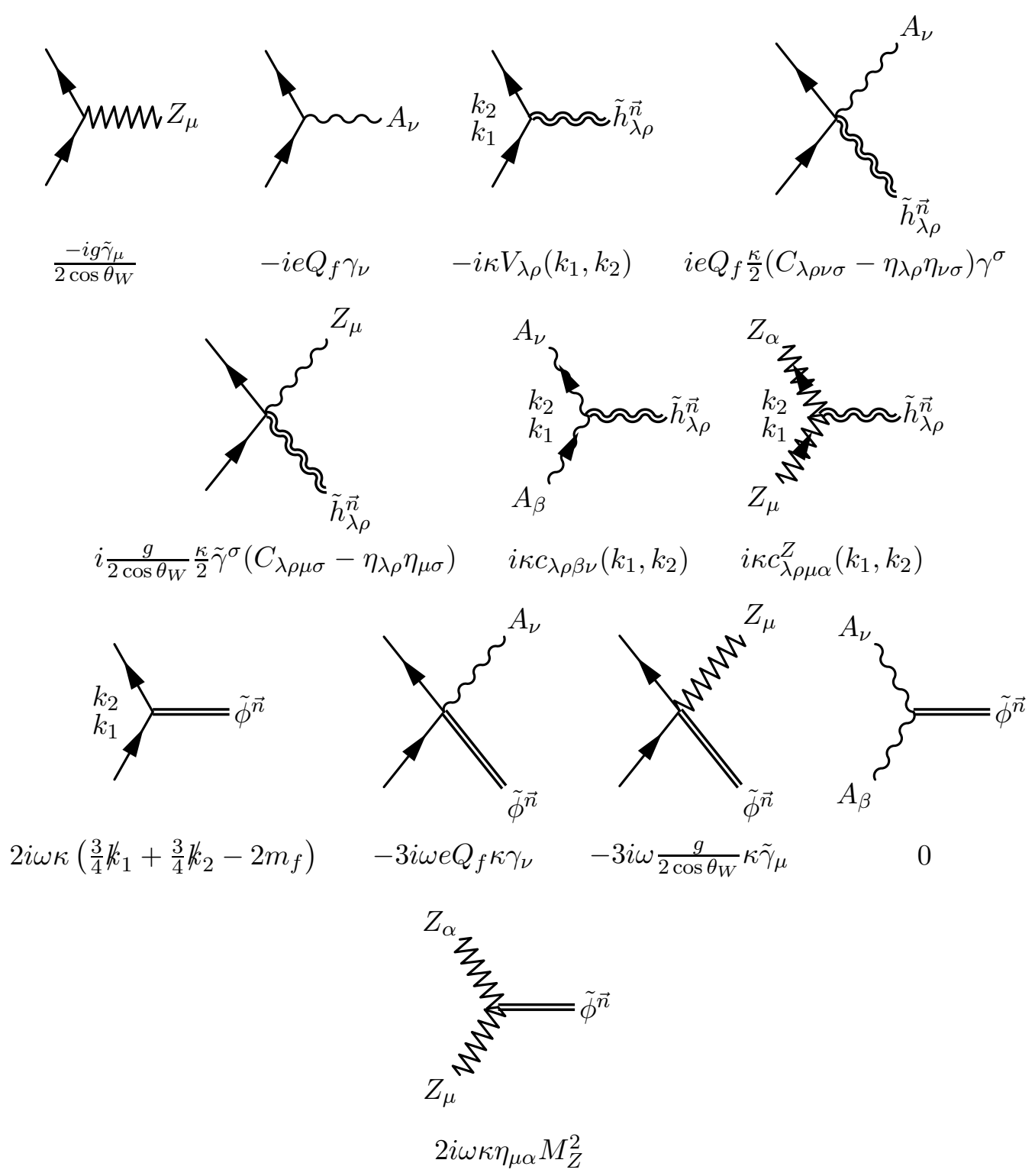

Figure 3: Feynman rules for vertices in fermion loop diagrams. The extra-dimensional indices are omitted from the vertices involving $\tilde{\phi}_{i j}^{\vec{n}}$ (each vertex gains a coefficient of $\delta_{i j}$ ). Symbols and tensors are defined in the main text. Arrows on bosonic lines indicate directions of flow of momenta.

and

$$
F_{\mu \nu}^{(\phi f)} \equiv \frac{\kappa e Q_{f} g}{2 \cos \theta_{W}} T_{\mu \nu}^{(\phi f)}
$$


we may write the contributions to the amplitudes from individual diagrams as

$$
\begin{aligned}
T_{\lambda \rho \mu \nu}^{(h f: \mathrm{a})} & =-\int \frac{d^{4} l}{(2 \pi)^{4}} \operatorname{Tr}\left[\tilde{\gamma}_{\mu} S(l-k) \gamma_{\nu} S(l) V_{\lambda \rho}(l, l+q) S(l+q)\right] \\
T_{\lambda \rho \mu \nu}^{(h f: \mathrm{b})} & =-\int \frac{d^{4} l}{(2 \pi)^{4}} \operatorname{Tr}\left[\tilde{\gamma}_{\mu} S(l-q) V_{\lambda \rho}(l, l-q) S(l) \gamma_{\nu} S(l+k)\right], \\
T_{\lambda \rho \mu \nu}^{(h f: \mathrm{c})} & =-i a_{\lambda \rho \nu \sigma} \Pi_{\mu}{ }^{\sigma}(p) \\
T_{\lambda \rho \mu \nu}^{(h f: \mathrm{d})} & =-i a_{\lambda \rho \mu \sigma} \Pi^{\sigma}{ }_{\nu}(k) \\
T_{\lambda \rho \mu \nu}^{(h f: \mathrm{e})} & =D^{\alpha \beta}(p) c_{\lambda \rho \beta \nu}(p, k) \Pi_{\mu \alpha}(p), \\
T_{\lambda \rho \mu \nu}^{(h f: \mathrm{f})} & =D_{Z}^{\alpha \beta}(k) c_{\lambda \rho \mu \alpha}^{Z}(p, k) \Pi_{\beta \nu}(k),
\end{aligned}
$$

and

$$
\begin{aligned}
T_{\mu \nu}^{(\phi f: \mathrm{a})} & =2 \omega \int \frac{d^{4} l}{(2 \pi)^{4}} \operatorname{Tr}\left[\tilde{\gamma}_{\mu} S(l-k) \gamma_{\nu} S(l)\left(\frac{3}{2} l+\frac{3}{4} \not l-2 m_{f}\right) S(l+q)\right], \\
T_{\mu \nu}^{(\phi f: \mathrm{b})} & =2 \omega \int \frac{d^{4} l}{(2 \pi)^{4}} \operatorname{Tr}\left[\tilde{\gamma}_{\mu} S(l-q)\left(\frac{3}{2} l-\frac{3}{4} \not l-2 m_{f}\right) S(l) \gamma_{\nu} S(l+k)\right], \\
T_{\mu \nu}^{(\phi f: \mathrm{c})} & =-3 i \omega \Pi_{\mu \nu}(p), \\
T_{\mu \nu}^{(\phi f: \mathrm{d})} & =-3 i \omega \Pi_{\mu \nu}(k), \\
T_{\mu \nu}^{(\phi f: \mathrm{e})} & =0 \\
T_{\mu \nu}^{(\phi f: \mathrm{f})} & =2 \omega \eta_{\mu \alpha} M_{Z}^{2} D_{Z}^{\alpha \beta}(k) \Pi_{\beta \nu}(k),
\end{aligned}
$$

where we have written

$$
a_{\lambda \rho \mu \nu} \equiv \eta_{\lambda \rho} \eta_{\mu \nu}-\frac{1}{2} \eta_{\lambda \mu} \eta_{\rho \nu}-\frac{1}{2} \eta_{\lambda \nu} \eta_{\rho \mu}
$$

and

$$
\Pi_{\mu \nu}(k) \equiv \int \frac{d^{4} l}{(2 \pi)^{4}} \operatorname{Tr}\left[\tilde{\gamma}_{\mu} S(l) \gamma_{\nu} S(l+k)\right],
$$

and have defined the propagators

$$
\begin{aligned}
S(l) & \equiv \frac{i\left(l+m_{f}\right)}{l^{2}-m_{f}^{2}}, \\
D^{\alpha \beta}(p) & \equiv \frac{-i \eta^{\alpha \beta}}{p^{2}}, \\
D_{Z}^{\alpha \beta}(k) & \equiv \frac{i}{k^{2}-M_{Z}^{2}}\left(-\eta^{\alpha \beta}+\frac{k^{\alpha} k^{\beta}}{M_{Z}^{2}}\right)
\end{aligned}
$$

for the fermion, photon and $\mathrm{Z}$ boson respectively (note that these are different from the definitions used in [1]). This amounts to a choice of unitary gauge for the electroweak sector. (We shall later need the propagator for the $\mathrm{W}$ boson, which we take to be that for the $\mathrm{Z}$ boson with $M_{Z} \rightarrow M_{W}$.) 


\section{W loop diagrams}

Figure 4 contains the diagrams for the $Z \rightarrow \gamma+\tilde{h}^{\vec{n}}$ process with a $\mathrm{W}$ boson in the loop, and figure 5 contains the diagrams for the $Z \rightarrow \gamma+\tilde{\phi}^{\vec{n}}$ process with a W boson in the loop. The Feynman rules for the vertices occurring in these diagrams additional to the fermionic loop case are given for reference in figure 6. Their derivation is given in reference [7]. Again, we

have abbreviated for legibility some of the tensor contributions to the vertex factors; the additional abbreviations used are

$$
\begin{aligned}
N_{\alpha \beta \gamma}\left(k_{1}, k_{2}, k_{3}\right) \equiv & \eta_{\beta \gamma}\left(k_{3}-k_{2}\right)_{\alpha}+\eta_{\gamma \alpha}\left(k_{1}-k_{3}\right)_{\beta}+\eta_{\alpha \beta}\left(k_{2}-k_{1}\right)_{\gamma}, \\
c_{\lambda \rho \beta \nu}^{W}\left(k_{1}, k_{2}\right) \equiv & c_{\lambda \rho \beta \nu}-M_{W}^{2} C_{\lambda \rho \beta \nu}, \\
d_{\lambda \rho \alpha \beta \nu}\left(k_{1}, k_{2}, k_{3}\right) \equiv & C_{\lambda \rho \alpha \beta}\left(k_{1}-k_{2}\right)_{\nu}+C_{\lambda \rho \alpha \nu}\left(k_{3}-k_{1}\right)_{\beta}+C_{\lambda \rho \beta \nu}\left(k_{2}-k_{3}\right)_{\alpha}+ \\
& +\left[\eta_{\lambda \alpha} \eta_{\beta \nu}\left(k_{2}-k_{3}\right)_{\rho}+\eta_{\lambda \beta} \eta_{\alpha \nu}\left(k_{3}-k_{1}\right)_{\rho}+\right. \\
& \left.\quad+\eta_{\lambda \nu} \eta_{\alpha \beta}\left(k_{1}-k_{2}\right)_{\rho}+(\lambda \leftrightarrow \rho)\right], \\
R_{\alpha \beta \mu \nu} \equiv & 2 \eta_{\alpha \beta} \eta_{\mu \nu}-\eta_{\alpha \mu} \eta_{\beta \nu}-\eta_{\alpha \nu} \eta_{\beta \mu}, \\
R_{\lambda \rho \alpha \beta \mu \nu} \equiv & \eta_{\lambda \rho} R_{\alpha \beta \nu \mu}-\eta_{\lambda \alpha} R_{\rho \beta \nu \mu}-\eta_{\lambda \beta} R_{\rho \alpha \nu \mu}-\eta_{\lambda \nu} R_{\rho \mu \alpha \beta}-\eta_{\lambda \mu} R_{\rho \nu \alpha \beta} .
\end{aligned}
$$

Writing $F_{\lambda \rho \mu \nu}^{(h W)}$ and $F_{\mu \nu}^{(\phi W)}$ to denote the contributions from the W boson loop diagrams to the off-shell amplitudes $F_{\lambda \rho \mu \nu}^{(h)}$ and $F_{\mu \nu}^{(\phi)}$, respectively, and this time absorbing constants by defining

$$
F_{\lambda \rho \mu \nu}^{(h W)} \equiv \kappa e g \cos \theta_{W} T_{\lambda \rho \mu \nu}^{(h W)}
$$

and

$$
F_{\mu \nu}^{(\phi W)} \equiv \kappa e g \cos \theta_{W} T_{\mu \nu}^{(\phi W)}
$$

we may write the contributions to the amplitudes from individual diagrams as

$$
\begin{aligned}
T_{\lambda \rho \mu \nu}^{(h W: \mathrm{a})}= & -\int \frac{d^{4} l}{(2 \pi)^{4}} N_{\alpha \beta \mu}(l,-l-p, p) D_{W}^{\alpha \tau}(l) c_{\lambda \rho \sigma \tau}^{W}(l+q, l) \times \\
& \times D_{W}^{\sigma \delta}(l+q) N_{\gamma \delta \nu}(l+p,-l-q,-k) D_{W}^{\gamma \beta}(l+p), \\
T_{\lambda \rho \mu \nu}^{(h W: \mathrm{b})}= & -\int \frac{d^{4} l}{(2 \pi)^{4}} N_{\alpha \beta \mu}(l-k,-l-q, p) D_{W}^{\alpha \tau}(l-k) \times \\
& \times N_{\sigma \tau \nu}(l,-l+k,-k) D_{W}^{\delta \sigma}(l) c_{\lambda \rho \gamma \delta}^{W}(l+q, l) D_{W}^{\beta \gamma}(l+q), \\
T_{\lambda \rho \mu \nu}^{(h W: \mathrm{c})}= & i \int \frac{d^{4} l}{(2 \pi)^{4}} N_{\alpha \beta \mu}(l,-l-p, p) D_{W}^{\alpha \tau}(l) d_{\lambda \rho \sigma \tau \nu}(l+p,-l,-k) D_{W}^{\sigma \beta}(l+p), \\
T_{\lambda \rho \mu \nu}^{(h W: \mathrm{d})}= & i \int \frac{d^{4} l}{(2 \pi)^{4}} d_{\lambda \rho \alpha \beta \mu}(l,-l-k, p) D_{W}^{\tau \alpha}(l) N_{\sigma \tau \nu}(l+k,-l,-k) D_{W}^{\beta \sigma}(l+k), \\
T_{\lambda \rho \mu \nu}^{(h W: \mathrm{e})}= & -D^{\gamma \delta}(p) c_{\lambda \rho \delta \nu}(p, k) \times \\
& \times \int \frac{d^{4} l}{(2 \pi)^{4}} N_{\alpha \beta \mu}(l,-l-p, p) D_{W}^{\tau \alpha}(l) N_{\sigma \tau \gamma}(l+p,-l,-p) D_{W}^{\beta \sigma}(l+p),
\end{aligned}
$$




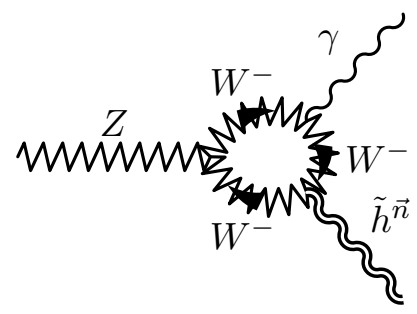

(a)

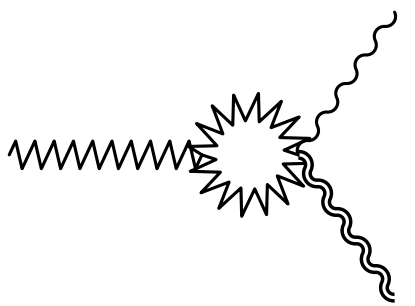

(c)

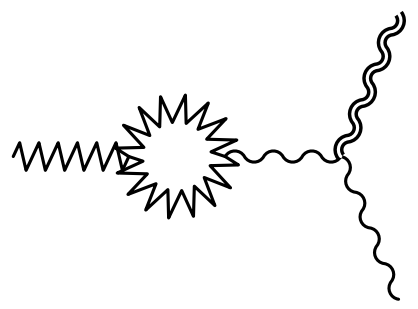

(e)

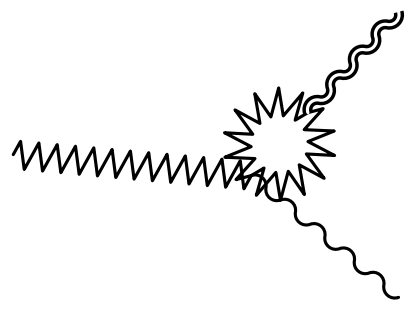

$(\mathrm{g})$

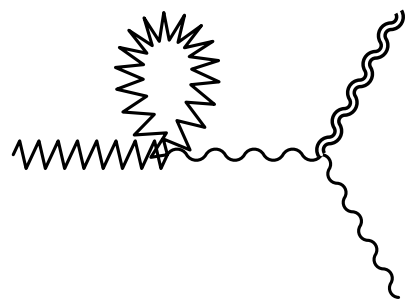

(i)

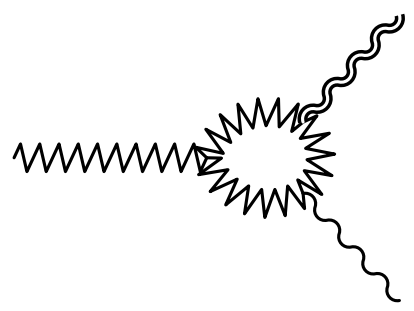

(b)

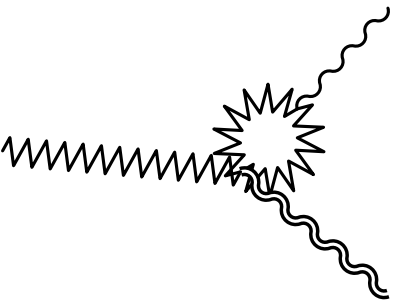

(d)

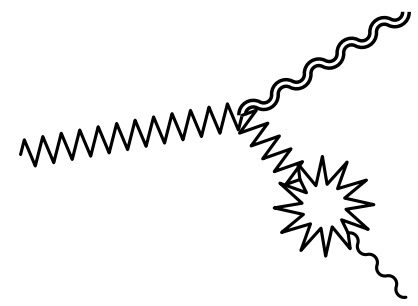

(f)

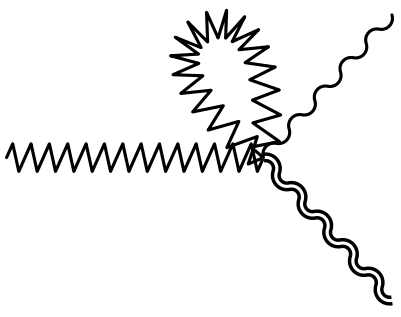

(h)

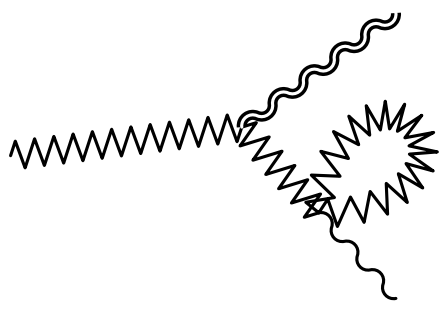

(j)

Figure 4: 1-loop diagrams for the process $Z \rightarrow \gamma+\tilde{h}^{\vec{n}}$ involving $\mathrm{W}$ bosons in the loop. We note that once one has defined a convention for charge and momentum flow in the loop, diagrams (a) and (b) must be treated as distinct. 


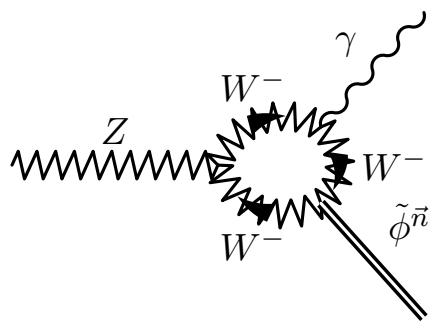

(a)

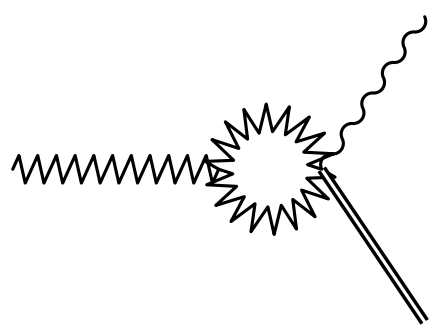

(c)

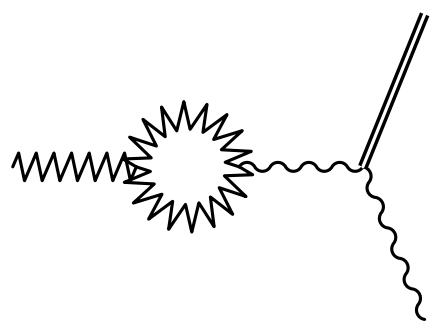

(e)

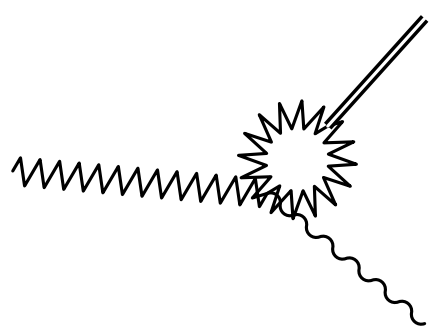

(g)

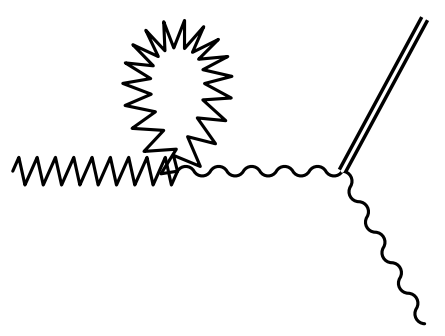

(i)

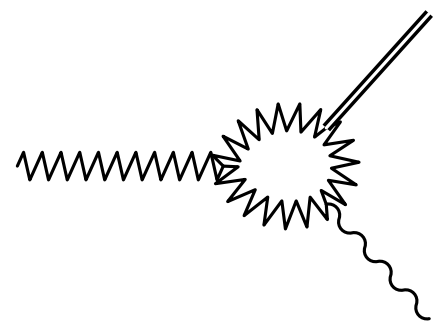

(b)

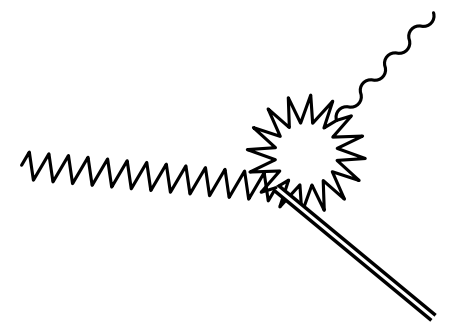

(d)

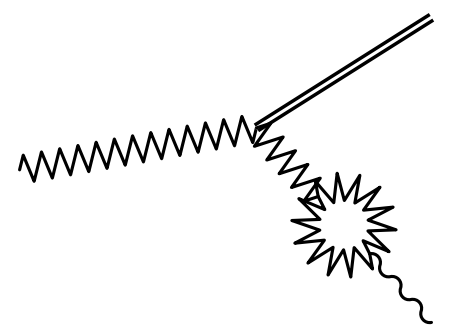

(f)

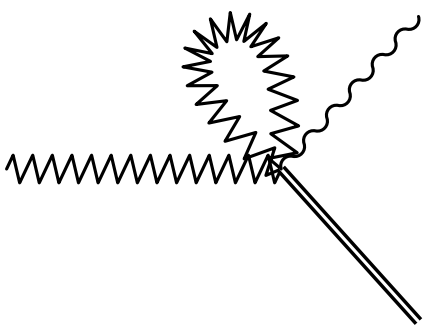

(h)

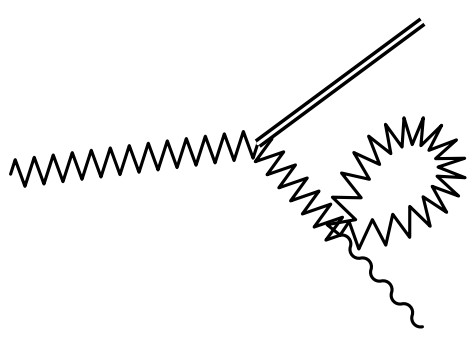

(j)

Figure 5: 1-loop diagrams for the process $Z \rightarrow \gamma+\tilde{\phi}^{\vec{n}}$ involving $\mathrm{W}$ bosons in the loop. 


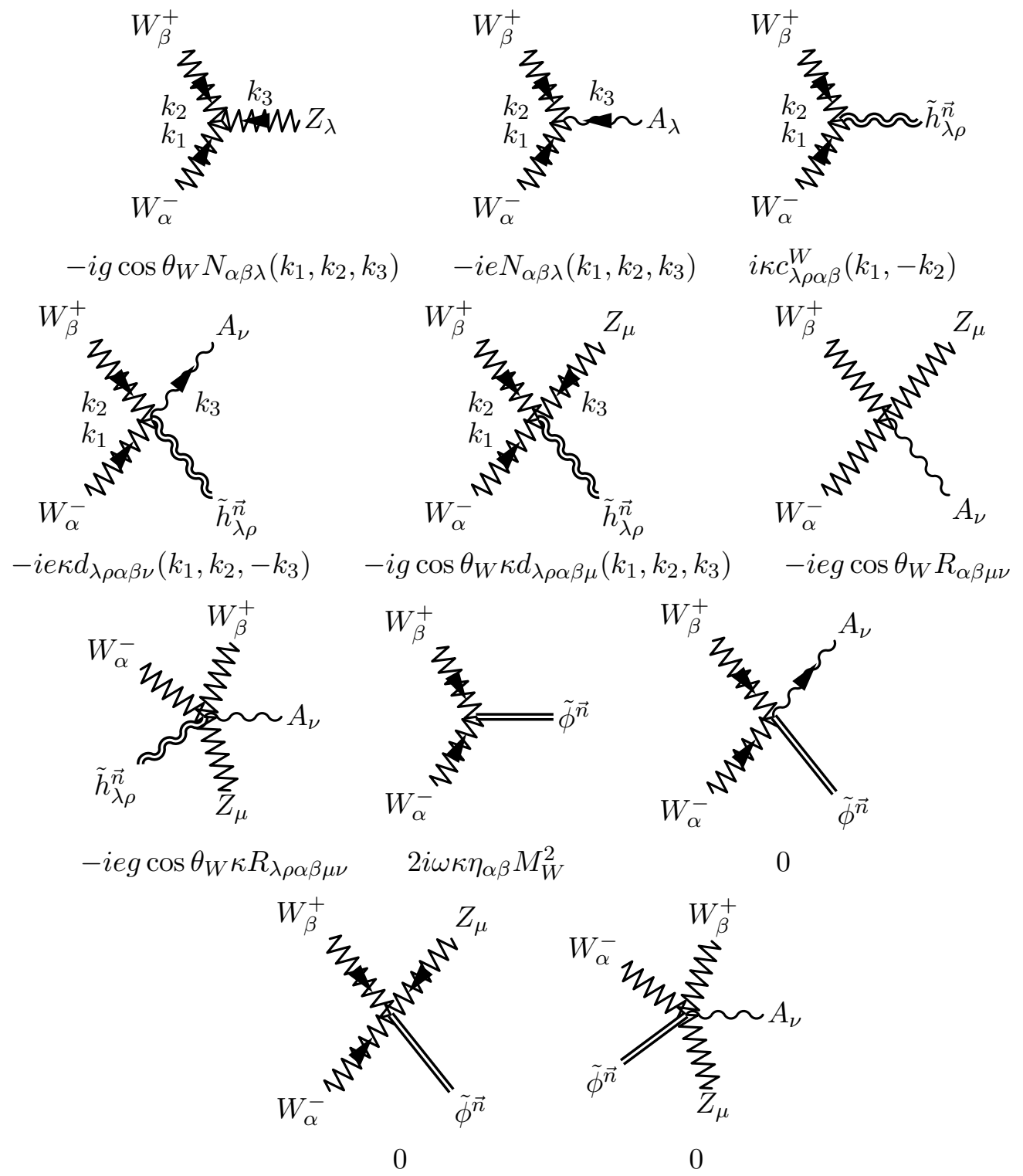

Figure 6: Extra Feynman rules for vertices in diagrams with $\mathrm{W}$ boson loops. Additional vertex functions are defined in the main text.

$$
\begin{aligned}
T_{\lambda \rho \mu \nu}^{(h W: \mathrm{f})}= & -c_{\lambda \rho \mu \alpha}^{Z}(p, k) D_{Z}^{\alpha \beta}(k) \times \\
& \times \int \frac{d^{4} l}{(2 \pi)^{4}} N_{\gamma \delta \beta}(l,-l-k, k) D_{W}^{\tau \gamma}(l) N_{\sigma \tau \nu}(l+k,-l,-k) D_{W}^{\delta \sigma}(l+k), \\
T_{\lambda \rho \mu \nu}^{(h W: \mathrm{g})}= & -i R_{\alpha \beta \mu \nu} \int \frac{d^{4} l}{(2 \pi)^{4}} D_{W}^{\tau \alpha}(l) c_{\lambda \rho \sigma \tau}^{W}(l+q, l) D_{W}^{\beta \sigma}(l+q), \\
T_{\lambda \rho \mu \nu}^{(h W: \mathrm{h})}= & -R_{\lambda \rho \alpha \beta \mu \nu} \int \frac{d^{4} l}{(2 \pi)^{4}} D_{W}^{\alpha \beta}(l),
\end{aligned}
$$




$$
\begin{aligned}
& T_{\lambda \rho \mu \nu}^{(h W: \mathrm{i})}=-i c_{\lambda \rho \alpha \nu}(p, k) D^{\alpha \beta}(p) R_{\sigma \tau \mu \beta} \int \frac{d^{4} l}{(2 \pi)^{4}} D_{W}^{\sigma \tau}(l), \\
& T_{\lambda \rho \mu \nu}^{(h W: \mathrm{j})}=-i c_{\lambda \rho \mu \alpha}^{Z}(p, k) D_{Z}^{\alpha \beta}(k) R_{\sigma \tau \beta \nu} \int \frac{d^{4} l}{(2 \pi)^{4}} D_{W}^{\sigma \tau}(l),
\end{aligned}
$$

and

$$
\begin{aligned}
T_{\mu \nu}^{(\phi W: \mathrm{a})}= & -2 \omega M_{W}^{2} \eta_{\sigma \tau} \int \frac{d^{4} l}{(2 \pi)^{4}} N_{\alpha \beta \mu}(l,-l-p, p) D_{W}^{\alpha \tau}(l) D_{W}^{\delta \sigma}(l+q) \times \\
& \times N_{\gamma \delta \nu}(l+p,-l-q,-k) D_{W}^{\beta \gamma}(l+p), \\
T_{\mu \nu}^{(\phi W: \mathrm{b})}= & -2 \omega M_{W}^{2} \eta_{\gamma \delta} \int \frac{d^{4} l}{(2 \pi)^{4}} N_{\alpha \beta \mu}(l-k,-l-q, p) D_{W}^{\alpha \tau}(l-k) \times \\
& \times N_{\sigma \tau \nu}(l,-l+k,-k) D_{W}^{\delta \sigma}(l) D_{W}^{\beta \gamma}(l+q), \\
T_{\mu \nu}^{(\phi W: \mathrm{f})}= & -2 \omega M_{Z}^{2} \eta_{\mu \alpha} D_{Z}^{\alpha \beta}(k) \times \\
& \times \int \frac{d^{4} l}{(2 \pi)^{4}} N_{\gamma \delta \beta}(l,-l-k, k) D_{W}^{\tau \gamma}(l) N_{\sigma \tau \nu}(l+k,-l,-k) D_{W}^{\delta \sigma}(l+k), \\
T_{\mu \nu}^{(\phi W: \mathrm{g})=} & -2 i \omega M_{W}^{2} \eta_{\sigma \tau} R_{\alpha \beta \mu \nu} \int \frac{d^{4} l}{(2 \pi)^{4}} D_{W}^{\tau \alpha}(l) D_{W}^{\beta \sigma}(l+q), \\
T_{\mu \nu}^{(\phi W: \mathrm{j})=} & -2 i \omega M_{Z}^{2} \eta_{\mu \alpha} R_{\sigma \tau \beta \nu} D_{Z}^{\alpha \beta}(k) \int \frac{d^{4} l}{(2 \pi)^{4}} D_{W}^{\tau \sigma}(l) ;
\end{aligned}
$$

we have omitted a number of lines corresponding to diagrams involving $\tilde{\phi}^{\vec{n}}$ production that evaluate to zero.

\section{Counterterm diagrams}

Because we are working at one loop using bare parameters, we must consider corrections that arise from a Standard Model Z- $\gamma$ mixing counterterm. It will turn out that such terms give a zero contribution (and this is why we work with bare parameters), and it is sufficient just to consider the general form that such terms take in order to demonstrate this.

Figure 7 contains the diagrams for the $Z \rightarrow \gamma+\tilde{h}^{\vec{n}}$ process that have a counterterm, and figure 8 contains the diagrams for the $Z \rightarrow \gamma+\tilde{\phi}^{\vec{n}}$ process that have a counterterm. We may divide the diagrams into two classes: those containing a two-point counterterm vertex and those containing a three-point counterterm vertex. The two-point counterterm vertex is the Z- $\gamma$ mixing counterterm that occurs in the Standard Model if one uses the tree-level diagonalization of the electroweak mixing matrix for one-loop calculations. The three-point counterterm vertex arises by considering the gravitational perturbation expansion about the Lagrangian term corresponding to the two-point counterterm vertex.

To derive the Feynman rules for the counterterm vertices in this regime, we need to consider the Lagrangian Z- $\gamma$ counterterm that arises from one-loop renormalization in the Standard Model [23]. The relevant terms in the bare Standard Model Lagrangian may be written as

$$
\mathcal{L}_{0 \mathrm{Z}, \gamma}=-\frac{1}{2} Z_{\mu}\left(-\eta^{\mu \nu} \partial^{2}+\partial^{\mu} \partial^{\nu}\right) Z_{\nu}-\frac{1}{2} A_{\mu}\left(-\eta^{\mu \nu} \partial^{2}+\partial^{\mu} \partial^{\nu}\right) A_{\nu}+\frac{1}{2} M_{Z}^{2} Z_{\mu} \eta^{\mu \nu} Z_{\nu},
$$




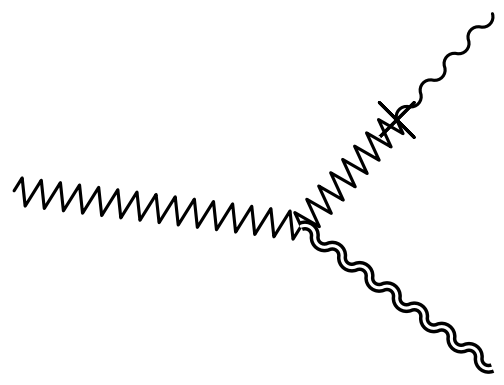

(a)

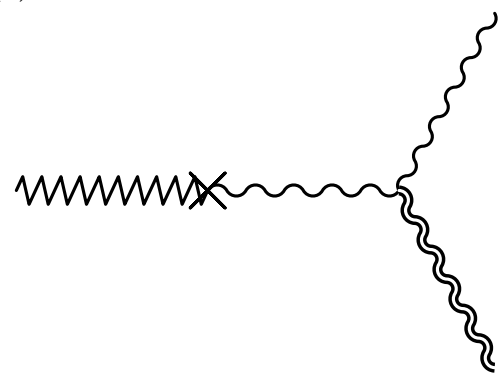

(c)

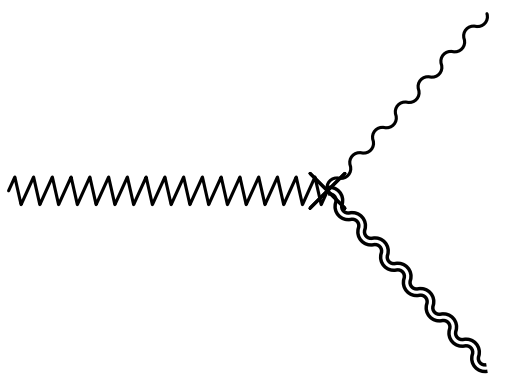

(b)

Figure 7: Diagrams for the process $Z \rightarrow \gamma+\tilde{h}^{\vec{n}}$ involving counterterm vertices.

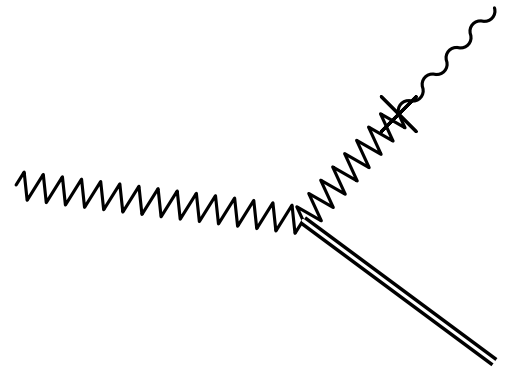

(a)

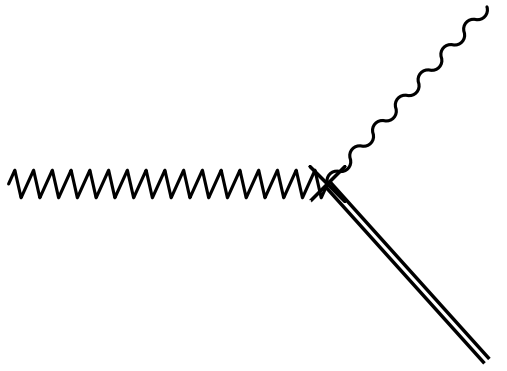

(b)

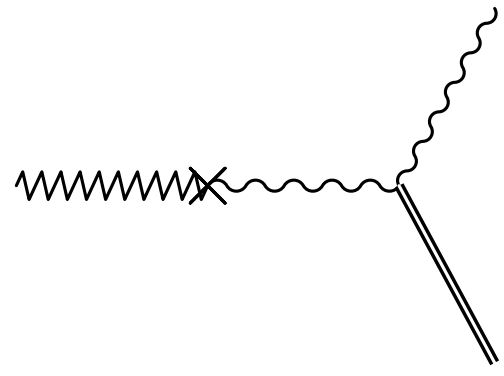

(c)

Figure 8: Diagrams for the process $Z \rightarrow \gamma+\tilde{\phi}^{\vec{n}}$ involving counterterm vertices.

and applying the renormalization

$$
\begin{aligned}
Z_{\mu} & \rightarrow Z_{Z Z}^{1 / 2} Z_{\mu}+Z_{Z A}^{1 / 2} A_{\mu}, \\
A_{\mu} & \rightarrow Z_{A Z}^{1 / 2} Z_{\mu}+Z_{A A}^{1 / 2} A_{\mu}, \\
M_{Z}^{2} & \rightarrow M_{Z}^{2}+\delta M_{Z}^{2}
\end{aligned}
$$


we obtain a mixing counterterm in the Lagrangian, which may be written

$$
\begin{aligned}
\mathcal{L}_{\mathrm{Z} \gamma}= & \left(Z_{Z Z}^{1 / 2} Z_{Z A}^{1 / 2}+Z_{A Z}^{1 / 2} Z_{A A}^{1 / 2}\right)\left[\partial_{\nu} Z^{\mu} \partial_{\mu} A^{\nu}-\eta_{\mu \nu} \partial^{\alpha} Z^{\mu} \partial_{\alpha} A^{\nu}\right]+ \\
& +\left(M_{Z}^{2}+\delta M_{Z}^{2}\right) Z_{Z Z}^{1 / 2} Z_{Z A}^{1 / 2} \eta_{\mu \nu} Z^{\mu} A^{\nu}
\end{aligned}
$$

We may read off from this that the Feynman rule for the two-point Z- $\gamma$ counterterm vertex with momentum $k$ passing through is [23]

$$
\begin{aligned}
& \left(\eta_{\mu \nu}-\frac{k_{\mu} k_{\nu}}{k^{2}}\right)\left[\left(M_{Z}^{2}+\delta M_{Z}^{2}\right)\left(Z_{Z Z}^{1 / 2} Z_{Z A}^{1 / 2}\right)-k^{2}\left(Z_{Z Z}^{1 / 2} Z_{Z A}^{1 / 2}+Z_{A Z}^{1 / 2} Z_{A A}^{1 / 2}\right)\right]+ \\
& +\frac{k_{\mu} k_{\nu}}{k^{2}}\left[\left(M_{Z}^{2}+\delta M_{Z}^{2}\right)\left(Z_{Z Z}^{1 / 2} Z_{Z A}^{1 / 2}\right)\right] .
\end{aligned}
$$

We write this contribution in the form

$$
A(k) \eta_{\mu \nu}+B k_{\mu} k_{\nu}
$$

where

$$
A(k)=\left(M_{Z}^{2}+\delta M_{Z}^{2}\right) Z_{Z Z}^{1 / 2} Z_{Z A}^{1 / 2}-k^{2}\left(Z_{Z Z}^{1 / 2} Z_{Z A}^{1 / 2}+Z_{A Z}^{1 / 2} Z_{A A}^{1 / 2}\right)
$$

and

$$
B=Z_{Z Z}^{1 / 2} Z_{Z A}^{1 / 2}+Z_{A Z}^{1 / 2} Z_{A A}^{1 / 2}
$$

For the three-point vertices, we must consider the gravitational coupling expansion of the metric. We may write the $O(\kappa)$ term of the expanded Lagrangian as [7]

$$
\mathcal{L}_{\kappa}=-\kappa \sum_{\vec{n}} \int d^{4} x\left(\tilde{h}^{\lambda \rho, \vec{n}} T_{\lambda \rho}+\omega \tilde{\phi}^{\vec{n}} T_{\lambda}^{\lambda}\right),
$$

where

$$
T_{\lambda \rho}=\left.\left(-\eta_{\lambda \rho} \mathcal{L}+2 \frac{\delta \mathcal{L}}{\delta g^{\lambda \rho}}\right)\right|_{g=\eta},
$$

and we have replaced Minkowski metric terms $\eta_{\mu \nu}$ in the Lagrangian with the perturbed metric $g_{\mu \nu}$. We find that the Z- $\gamma$ mixing terms in the energy-momentum tensor are

$$
\begin{aligned}
T_{\lambda \rho}^{(\mathrm{Z} \gamma)}= & \left(Z_{Z Z}^{1 / 2} Z_{Z A}^{1 / 2}+Z_{A Z}^{1 / 2} Z_{A A}^{1 / 2}\right) \times \\
\times & {\left[\eta_{\mu \nu} \eta_{\lambda \rho} \partial^{\alpha} Z^{\mu} \partial_{\alpha} A^{\nu}-\eta_{\lambda \rho} \partial_{\nu} Z^{\mu} \partial_{\mu} A^{\nu}-\eta_{\mu \lambda} \eta_{\nu \rho} \partial^{\alpha} Z^{\mu} \partial_{\alpha} A^{\nu}-\right.} \\
& -\eta_{\mu \rho} \eta_{\nu \lambda} \partial^{\alpha} Z^{\mu} \partial_{\alpha} A^{\nu}-\eta_{\mu \nu} \partial_{\lambda} Z^{\mu} \partial_{\rho} A^{\nu}-\eta_{\mu \nu} \partial_{\rho} Z^{\mu} \partial_{\lambda} A^{\nu}+ \\
& \left.+\eta_{\mu \lambda} \partial_{\nu} Z^{\mu} \partial_{\rho} A^{\nu}+\eta_{\mu \rho} \partial_{\nu} Z^{\mu} \partial_{\lambda} A^{\nu}+\eta_{\rho \nu} \partial_{\lambda} Z^{\mu} \partial_{\mu} A^{\nu}+\eta_{\lambda \nu} \partial_{\rho} Z^{\mu} \partial_{\mu} A^{\nu}\right]+ \\
+ & \left(M_{Z}^{2}+\delta M_{Z}^{2}\right) Z_{Z Z}^{1 / 2} Z_{Z A}^{1 / 2}\left[\eta_{\mu \lambda} \eta_{\rho \nu}+\eta_{\mu \rho} \eta_{\lambda \nu}-\eta_{\mu \nu} \eta_{\lambda \rho}\right] Z^{\mu} A^{\nu}
\end{aligned}
$$




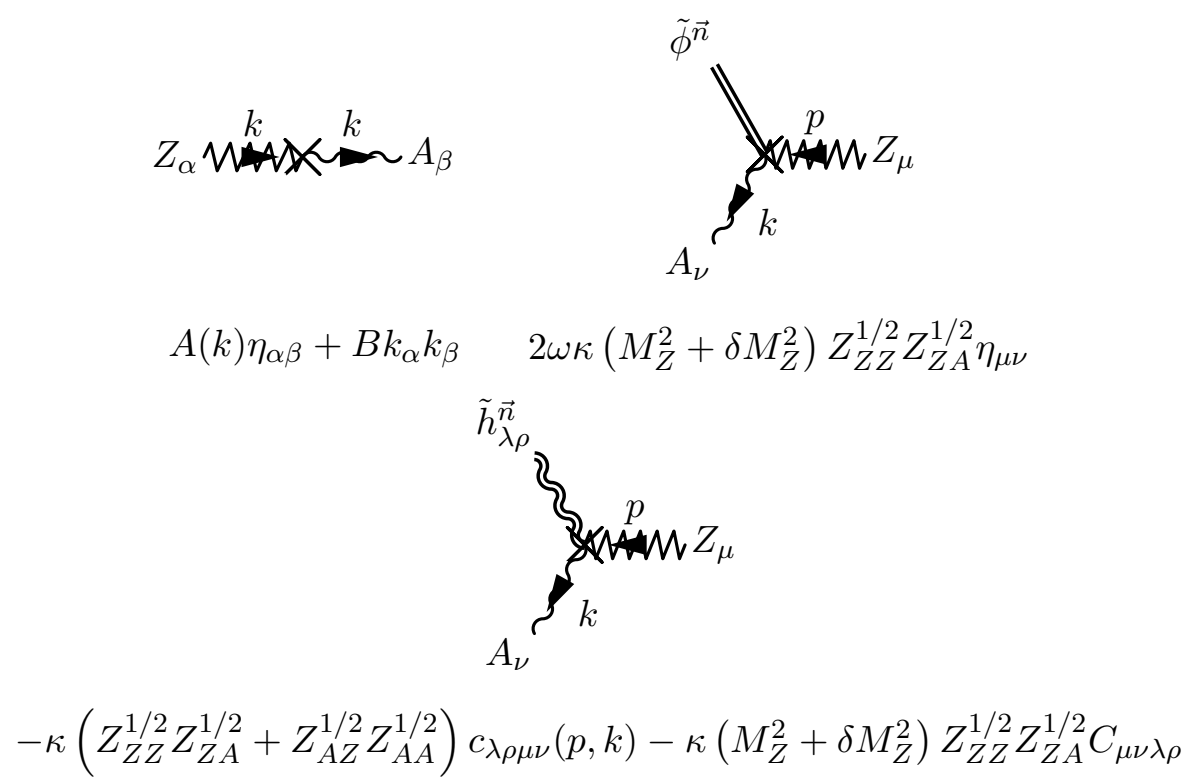

Figure 9: Extra Feynman rules for counterterm vertices. Abbreviations are defined in the main text.

with trace

$$
T_{\lambda}^{\lambda}=-2\left(M_{Z}^{2}+\delta M_{Z}^{2}\right) Z_{Z Z}^{1 / 2} Z_{Z A}^{1 / 2} \eta_{\mu \nu} Z^{\mu} A^{\nu}
$$

This yields a Feynman rule for the vertex $Z^{\mu}(p) A^{\nu}(k) \tilde{h}^{\lambda \rho, \vec{n}}(q)$ of

$$
-\kappa\left(Z_{Z Z}^{1 / 2} Z_{Z A}^{1 / 2}+Z_{A Z}^{1 / 2} Z_{A A}^{1 / 2}\right) c_{\lambda \rho \mu \nu}(p, k)-\kappa\left(M_{Z}^{2}+\delta M_{Z}^{2}\right) Z_{Z Z}^{1 / 2} Z_{Z A}^{1 / 2} C_{\mu \nu \lambda \rho},
$$

where $C_{\mu \nu \lambda \rho}$ and $c_{\lambda \rho \mu \nu}(p, k)$ are defined in equations (2.1) and (2.2) respectively, and a Feynman rule for the vertex $Z^{\mu}(p) A^{\nu}(k) \tilde{\phi}^{\vec{n}}(q)$ of

$$
2 \omega \kappa\left(M_{Z}^{2}+\delta M_{Z}^{2}\right) Z_{Z Z}^{1 / 2} Z_{Z A}^{1 / 2} \eta_{\mu \nu}
$$

We note for future reference that the form of the Z- $\gamma-\tilde{h}^{n}$ vertex is such that there is no term in which there are four momenta all carrying Lorentz indices (this will be important in showing that the counterterms give no contribution to the amplitude).

The additional Feynman rules are summarized in figure 9 .

With these Feynman rules, and writing $F_{\lambda \rho \mu \nu}^{(h \times)}$ and $F_{\mu \nu}^{(\phi \times)}$ to denote the contributions from the counterterm diagrams to the off-shell amplitudes $F_{\lambda \rho \mu \nu}^{(h)}$ and $F_{\mu \nu}^{(\phi)}$, respectively, we may write the contributions from individual diagrams to the amplitudes as

$$
\begin{aligned}
& F_{\lambda \rho \mu \nu}^{(h \times: \mathrm{a})}=\kappa c_{\lambda \rho \mu \alpha}^{Z}(p, k) D_{Z}^{\alpha \beta}(k)\left[A(k) \eta_{\beta \nu}+B k_{\beta} k_{\nu}\right] \\
& F_{\lambda \rho \mu \nu}^{(h \times \mathrm{b})}=i \kappa\left[\left(Z_{Z Z}^{1 / 2} Z_{Z A}^{1 / 2}+Z_{A Z}^{1 / 2} Z_{A A}^{1 / 2}\right) c_{\lambda \rho \mu \nu}(p, k)+\left(M_{Z}^{2}+\delta M_{Z}^{2}\right) Z_{Z Z}^{1 / 2} Z_{Z A}^{1 / 2} C_{\mu \nu \lambda \rho}\right], \\
& F_{\lambda \rho \mu \nu}^{(h \times: \mathrm{c})}=\kappa c_{\lambda \rho \beta \nu}(p, k) D^{\alpha \beta}(p)\left[A(p) \eta_{\mu \alpha}+B p_{\mu} p_{\alpha}\right]
\end{aligned}
$$


and

$$
\begin{aligned}
& F_{\mu \nu}^{(\phi \times: \mathrm{a})}=2 \omega \kappa \eta_{\mu \alpha} M_{Z}^{2} D_{Z}^{\alpha \beta}(k)\left[A(k) \eta_{\beta \nu}+B k_{\beta} k_{\nu}\right] \\
& F_{\mu \nu}^{(\phi \times: \mathrm{b})}=-2 i \omega \kappa\left(M_{Z}^{2}+\delta M_{Z}^{2}\right) Z_{Z Z}^{1 / 2} Z_{Z A}^{1 / 2} \eta_{\mu \nu} \\
& F_{\mu \nu}^{(\phi \times: \mathrm{c})}=0 .
\end{aligned}
$$

\section{General forms of the amplitudes}

We now derive general forms that must be taken by the amplitudes we are calculating; these forms allow simplification of the calculation. The argument for the decay involving a spin-2 graviton excitation is that of Nieves and Pal [1]; the argument for the decay involving a spin-0 graviton excitation is essentially the first part of the argument for the spin-2 case, and is given first as it is more straightforward.

The arguments rely upon Ward-Takahashi identities that are consequences of electromagnetic and gravitational gauge invariance. This gauge invariance is shown in the next section for each of the sets of fermion loop diagrams, W loop diagrams and counterterm diagrams separately.

\subsection{Decay into spin-0 excitation and photon}

Electromagnetic gauge invariance implies conservation of the electromagnetic current, which (transforming to momentum space) gives

$$
k^{\nu} F_{\mu \nu}^{(\phi)}(q, k)=0 .
$$

We may expand $F_{\mu \nu}^{(\phi)}$ about $k=0$, writing

$$
F_{\mu \nu}^{(\phi)}=\mathcal{T}_{\mu \nu}^{0}+k^{\alpha} \mathcal{T}_{\mu \nu \alpha}^{1},
$$

with $\mathcal{T}_{\mu \nu}^{0}$ independent of $k$. Equation (5.1) then implies that

$$
\begin{aligned}
k^{\nu} \mathcal{T}_{\mu \nu}^{0} & =0, \\
k^{\nu} k^{\alpha} \mathcal{T}_{\mu \nu \alpha}^{1} & =0,
\end{aligned}
$$

and since this is true for all $k$ with $\left|k_{0}\right| \leq M_{Z}$ in the centre of mass frame and $k^{2}=0$, we can deduce that

$$
\mathcal{T}_{\mu \nu}^{0}=0
$$

and

$$
\mathcal{T}_{\mu \nu \alpha}^{1}=-\mathcal{T}_{\mu \alpha \nu}^{1} .
$$

This means we may write the on-shell amplitude (1.19) as

$$
\mathcal{M}^{(\phi)}(q, k)=\varepsilon_{\mathrm{Z}}^{\mu}(p) f^{\nu \alpha *} t_{\mu \nu \alpha},
$$


where

$$
f_{\nu \alpha} \equiv k_{\nu} \varepsilon_{\alpha}-k_{\alpha} \varepsilon_{\nu}
$$

and

$$
t_{\mu \nu \alpha}=-t_{\mu \alpha \nu}
$$

Recalling equations (1.9) and (1.14) $\left(\varepsilon^{\nu}(k) k_{\nu}=0\right.$ and $\left.k^{2}=0\right)$, along with the momentum conservation relation $p=k+q$, and considering terms that could be contained in $t_{\mu \nu \alpha}$, we see that the only terms contributing to the amplitude are ones not involving a Levi-Civita tensor, of the form

$$
\text { scalar } \times \varepsilon_{\mathrm{Z}}^{\mu}(p)\left(k^{\nu} \varepsilon^{\alpha *}-k^{\alpha} \varepsilon^{\nu *}\right)\left(\eta_{\mu \nu} q_{\alpha}-\eta_{\mu \alpha} q_{\nu}\right),
$$

and ones involving a Levi-Civita tensor, of the form

$$
\text { scalar } \times \varepsilon_{\mathrm{Z}}^{\mu}(p) \epsilon^{\nu \alpha \gamma \delta}\left(k_{\gamma} \varepsilon_{\delta *}-k_{\delta} \varepsilon_{\gamma *}\right)\left(\eta_{\mu \nu} q_{\alpha}-\eta_{\mu \alpha} q_{\nu}\right) .
$$

Relabelling indices so that the polarisation tensors may be written as common coefficients of the overall amplitude, we may rewrite the above as

$$
F_{\mu \nu}^{(\phi)}=\left(k_{\mu} q_{\nu}-k \cdot q \eta_{\mu \nu}\right) F^{(\phi)}+\left(\epsilon_{\mu \nu \alpha \beta} q^{\alpha} k^{\beta}\right) F_{1}^{(\phi)},
$$

where $F^{(\phi)}$ and $F_{1}^{(\phi)}$ are Lorentz scalars.

\subsection{Decay into spin-2 excitation and photon}

The argument in the case of decay involving a spin-2 graviton excitation may be viewed as an extension of the case involving a spin-0 graviton excitation. It is given in full in reference [1] for the case of a massless graviton, and is sketched here.

Similarly to the spin-0 case, we may write

$$
F_{\lambda \rho \mu \nu}=\mathcal{T}_{\lambda \rho \mu \nu}^{0}+k^{\alpha} \mathcal{T}_{\lambda \rho \mu \nu \alpha}^{1}
$$

and we may use the condition $k^{\nu} F_{\lambda \rho \mu \nu}$ to deduce that we may write the on-shell amplitude (1.18) in the form

$$
\mathcal{M}^{(h)}(q, k)=\mathcal{E}^{\lambda \rho}(q) \varepsilon_{\mathrm{Z}}^{\mu}(p) f^{\nu \alpha *} t_{\lambda \rho \mu \nu \alpha},
$$

where $f_{\nu \alpha}$ is as defined in equation (5.8).

We may also use gravitational gauge invariance to derive a manner of writing the amplitude, in a similar fashion to the way we have used electromagnetic invariance (although the details are complicated by the extra Lorentz index in the gravitational case).

Writing

$$
\mathcal{M}^{(h)}=\mathcal{E}^{\lambda \rho}(q) j_{\lambda \rho}(q, k),
$$

and expanding

$$
j_{\lambda \rho}=j_{\lambda \rho}^{0}+q^{\sigma} j_{\lambda \rho \sigma}^{1}+q^{\tau} q^{\sigma} j_{\lambda \rho \sigma \tau}^{2}
$$


where $j_{\lambda \rho}^{0}$ and $j_{\lambda \rho \sigma}^{1}$ are independent of $q$, we may use the conditions

$$
q^{\lambda} j_{\lambda \rho}=0, \quad q^{\rho} j_{\lambda \rho}=0
$$

(following from conservation of the energy-momentum tensor) to deduce

$$
\begin{gathered}
j_{\lambda \rho}^{0}=0, \\
j_{\lambda \rho \sigma}^{1}=0 .
\end{gathered}
$$

In addition, $j_{\lambda \rho \sigma \tau}^{2}$ has a number of symmetries arising from its definition (namely $\lambda \leftrightarrow \rho$ symmetry, $\sigma \leftrightarrow \tau$ symmetry and antisymmetry under interchange of either of $(\lambda, \rho)$ with either of $(\sigma, \tau))$. We also note that no term in the expansion of $j_{\lambda \rho \sigma \tau}^{2}$ contains a $q_{\lambda}$ or $q_{\rho}$ term, because such terms vanish on contraction with the gravitational polarisation tensor (equation (1.11)). Further, we need not consider terms in $q_{\sigma}$ or $q_{\tau}$, since $q^{2}=m_{\vec{n}}^{2}$, and so such terms reduce to lower order terms in equation (5.16). Combining these properties with the expression for the form of the amplitude obtained from consideration of the electromagnetic gauge invariance yields a general form of the amplitude that may be written as

$$
\begin{aligned}
F_{\lambda \rho \mu \nu}^{(h)}=\{ & \left(k_{\lambda} q_{\nu}-k \cdot q \eta_{\nu \lambda}\right)\left(k_{\rho} q_{\mu}-k \cdot q \eta_{\mu \rho}\right) F^{(h)}+\epsilon_{\lambda \nu \alpha \beta} q^{\alpha} k^{\beta}\left(k_{\rho} q_{\mu}-k \cdot q \eta_{\mu \rho}\right) F_{1}^{(h)}+ \\
& \left.+\left(k_{\lambda} q_{\nu}-k \cdot q \eta_{\nu \lambda}\right) \epsilon_{\rho \mu \alpha \beta} q^{\alpha} k^{\beta} F_{2}^{(h)}\right\}+(\lambda \leftrightarrow \rho) .
\end{aligned}
$$

\section{Transversality conditions}

In order to apply to any set of diagrams the arguments presented in section 5 regarding the general forms of the amplitudes, we must establish that if we take the contribution given by the set of diagrams to the amplitude and contract it with the photon momentum, this yields zero. (In the case of the decays involving a spin-2 graviton excitation, we also need to establish that contraction with the graviton momentum yields zero.)

\subsection{Electromagnetic transversality: spin-2 case}

We begin by considering the case of decays involving production of a spin-2 graviton excitation. In the spin- 2 case, the contributions to the amplitude of the diagrams involving a fermion loop and of the diagrams involving a $\mathrm{W}$ boson loop are essentially the same as the contributions given in [1]. We repeat here for completeness an outline of the derivation of the transversality results in those cases. The counterterm diagrams are not considered explicitly in [1], and we present more fully the argument for these diagrams.

\subsubsection{Fermion loop diagrams}

Using the identity ${ }^{2}$

$$
k^{\nu} S(l) \gamma_{\nu} S(l+k)=i S(l)-i S(l+k)
$$

\footnotetext{
${ }^{2}$ The identities differ from those given in [1] by factors of $i$ owing to the different propagator definitions.
} 
with equation (2.22) implies that

$$
k^{\nu} \Pi_{\mu \nu}(k)=0,
$$

from which, using equations $(2.12)$ and $(2.14)$, it follows that

$$
k^{\nu} T_{\lambda \rho \mu \nu}^{(h f: \mathrm{d})}=0, \quad k^{\nu} T_{\lambda \rho \mu \nu}^{(h f: \mathrm{f})}=0 .
$$

Using the definition of equation (2.2), we also have

$$
k^{\nu} c_{\lambda \rho \beta \nu}(p, k)=0
$$

from which it follows that

$$
k^{\nu} T_{\lambda \rho \mu \nu}^{(h f: \mathrm{e})}=0 .
$$

We can show that

$$
k^{\nu} T_{\lambda \rho \mu \nu}^{(h f: \mathrm{a})}+k^{\nu} T_{\lambda \rho \mu \nu}^{(h f: \mathrm{b})}+k^{\nu} T_{\lambda \rho \mu \nu}^{(h f: \mathrm{c})}=0,
$$

by using equation (6.1) on the sum $k^{\nu} T_{\lambda \rho \mu \nu}^{(h f: \mathrm{a})}+k^{\nu} T_{\lambda \rho \mu \nu}^{(h f: \mathrm{b})}$ and writing the resultant term so that the only propagator terms are $S(l)$ and $S(l+p)$, and then using the Ward-Takahashi identity

$$
V_{\lambda \rho}(l+q, l)-V_{\lambda \rho}(l+p, l+k)=a_{\lambda \rho \alpha \beta} \gamma^{\alpha} k^{\beta}
$$

This establishes that

$$
k^{\nu} T_{\lambda \rho \mu \nu}^{(h f)}=0
$$

\subsubsection{W loop diagrams}

Electromagnetic transversality of the $\mathrm{W}$ loop diagrams contributing to the spin-2 excitation producing decay may be demonstrated by using a number of Ward-Takahashi identities. The identities

$$
\begin{aligned}
r^{\gamma} D_{W}^{\alpha \sigma}(l+r) N_{\alpha \beta \gamma}(l+r,-l,-r) D_{W}^{\beta \tau}(l) & =i D_{W}^{\sigma \tau}(l)-i D_{W}^{\sigma \tau}(l+r), \\
c_{\lambda \rho \alpha \beta}^{W}(l+s, l)-c_{\lambda \rho \alpha \beta}^{W}(l+r+s, l+r) & =r^{\gamma} d_{\lambda \rho \alpha \beta \gamma}(l+r+s,-l,-r),
\end{aligned}
$$

and

$$
N_{\alpha, \beta, \gamma}(l-r,-l-s, r+s)-N_{\alpha, \beta, \gamma}(l,-l-r-s, r+s)=r^{\delta} R_{\alpha \beta \gamma \delta},
$$

together with equation (6.4), can be used with equations $(3.8)$ to $(3.17)$ to deduce that

$$
\begin{aligned}
k^{\nu} T_{\lambda \rho \mu \nu}^{(h W: \mathrm{a})}+k^{\nu} T_{\lambda \rho \mu \nu}^{(h W: \mathrm{b})}+k^{\nu} T_{\lambda \rho \mu \nu}^{(h W: \mathrm{c})}+k^{\nu} T_{\lambda \rho \mu \nu}^{(h W: \mathrm{g})} & =0, \\
k^{\nu} T_{\lambda \rho \mu \nu}^{(h W: \mathrm{d})}+k^{\nu} T_{\lambda \rho \mu \nu}^{(h W: \mathrm{h})} & =0, \\
k^{\nu} T_{\lambda \rho \mu \nu}^{(h W: \mathrm{e})} & =0, \\
k^{\nu} T_{\lambda \rho \mu \nu}^{(h W: \mathrm{f})}+k^{\nu} T_{\lambda \rho \mu \nu}^{(h W: \mathrm{j})} & =0,
\end{aligned}
$$


and

$$
k^{\nu} T_{\lambda \rho \mu \nu}^{(h W: \mathrm{i})}=0
$$

and therefore that

$$
k^{\nu} T_{\lambda \rho \mu \nu}^{(h W)}=0
$$

\subsubsection{Counterterm diagrams}

Using equation (6.4) with the definition of equation (4.18) implies that

$$
k^{\nu} F_{\lambda \rho \mu \nu}^{(h \times: \mathrm{c})}=0 .
$$

Equation (6.4) also allows us to simplify the forms of $k^{\nu} F_{\lambda \rho \mu \nu}^{(h \times: \mathrm{a})}$ and $k^{\nu} F_{\lambda \rho \mu \nu}^{(h \times \mathrm{b})}$. If we expand the definitions of $c_{\lambda \rho \mu \alpha}^{Z}(p, k)$ and $D_{Z}^{\alpha \beta}(k)$ from equations (2.3) and (2.25) respectively, this yields

$$
k^{\nu} F_{\lambda \rho \mu \nu}^{(h \times: \mathrm{a})}=\kappa A(k)\left(k_{\mu} \eta_{\lambda \rho}-k_{\lambda} \eta_{\mu \rho}-k_{\rho} \eta_{\mu \lambda}\right)=-k^{\nu} F_{\lambda \rho \mu \nu}^{(h \times: \mathrm{b})},
$$

from which we deduce that

$$
k^{\nu} F_{\lambda \rho \mu \nu}^{(h \times: \mathrm{a})}+k^{\nu} F_{\lambda \rho \mu \nu}^{(h \times: \mathrm{b})}+k^{\nu} F_{\lambda \rho \mu \nu}^{(h \times: \mathrm{c})}=0 .
$$

\subsection{Gravitational transversality: spin-2 case}

\subsubsection{Fermion loop diagrams}

Gravitational transversality of the fermion loop diagrams contributing to the spin-2 graviton excitation production process may be seen through use of the identities

$$
\begin{aligned}
S(l) q^{\lambda} V_{\lambda \rho}(l+q, l) S(l+q)= & i\left(l_{\rho}+q_{\rho}\right) S(l+q)-i l_{\rho} S(l)+ \\
& +\frac{i}{8}\left(\gamma_{\rho} \not l-\not q \gamma_{\rho}\right) S(l+q)-\frac{i}{8} S(l)\left(\gamma_{\rho} \not-\not q \gamma_{\rho}\right)
\end{aligned}
$$

and

$$
\gamma_{\alpha} \gamma_{\beta} \gamma_{\rho}+\gamma_{\rho} \gamma_{\beta} \gamma_{\alpha}=2\left(\eta_{\alpha \beta} \gamma_{\rho}+\eta_{\beta \rho} \gamma_{\alpha}-\eta_{\alpha \rho} \gamma_{\beta}\right),
$$

together with the momentum conditions and with changes in the integration variable in some of the loop momenta integrations. Fuller details may be found in reference [1].

\subsubsection{W loop diagrams}

If we use cyclic identity

$$
R_{\alpha \beta \gamma \delta}+R_{\beta \gamma \alpha \delta}+R_{\gamma \alpha \beta \delta}=0
$$

together with the identities

$$
N_{\alpha \beta \gamma}(l,-l-s, s)-N_{\alpha \beta \gamma}(l,-l-s+r, s-r)=r^{\delta} R_{\beta \gamma \alpha \delta}
$$


and

$$
N_{\alpha \beta \gamma}(l,-l-s, s)-N_{\alpha \beta \gamma}(l-r,-l-s, r+s)=r^{\delta} R_{\gamma \alpha \beta \delta},
$$

the identity of equation (6.10) and the identity

$$
\begin{aligned}
q^{\lambda} D_{W}^{\alpha \sigma}(l+q) c_{\lambda \rho \alpha \beta}^{W} D_{W}^{\beta \tau}= & i\left(l_{\rho}+q_{\rho}\right) D_{W}^{\sigma \tau}(l+q)-i l_{\rho} D_{W}^{\sigma \tau}(l)- \\
& -i \eta_{\rho \gamma}\left(q^{\sigma} D_{W}^{\tau \gamma}(l)+q^{\tau} D_{W}^{\sigma \gamma}(l+q)\right),
\end{aligned}
$$

then it is possible to derive the result

$$
q^{\lambda} T_{\lambda \rho \mu \nu}^{(h W)}=0
$$

More details of the manipulations involved may be found in reference [1].

\subsubsection{Counterterm diagrams}

Gravitational transversality of the counterterm diagrams may be shown simply by contracting each of the diagrams with $q^{\lambda}$ and summing the three resultant expressions, if one uses the on-shell momentum conditions for the $\mathrm{Z}$ boson and the photon (equations (1.13) and (1.14) ) 24.

\subsection{Electromagnetic transversality: spin-0 case}

We now turn to demonstrating electromagnetic transversality of the separate classes of diagrams in the case of decays involving spin-0 graviton excitation production. This is more straightforward than the spin-2 case, because there are fewer non-zero diagrams to consider. (In addition, we only need to demonstrate electromagnetic transversality to establish that the contributions from the classes of diagrams are of the general form given in equation (5.12): there is no corresponding gravitational transversality condition to be satisfied). We use some of the identities from the spin-2 case.

\subsubsection{Fermion loop diagrams}

Using equation (6.2) with equations (2.18) and (2.20) implies that

$$
k^{\nu} T_{\mu \nu}^{(\phi f: \mathrm{d})}=0, \quad k^{\nu} T_{\mu \nu}^{(\phi f: \mathrm{f})}=0 .
$$

In addition, we can use equation (6.1) with equations 2.15$)$ and $(2.16)$ to write

$$
k^{\nu} T_{\mu \nu}^{(\phi f: \mathrm{a})}=2 i \omega \int \frac{d^{4} l}{(2 \pi)^{4}} \operatorname{Tr}\left[\tilde{\gamma}_{\mu}\{S(l-k)-S(l)\}\left(\frac{3}{2} \not l+\frac{3}{4} \not l-2 m_{f}\right) S(l+q)\right]
$$

and

$$
k^{\nu} T_{\mu \nu}^{(\phi f: \mathrm{b})}=2 i \omega \int \frac{d^{4} l}{(2 \pi)^{4}} \operatorname{Tr}\left[\tilde{\gamma}_{\mu} S(l-q)\left(\frac{3}{2} l-\frac{3}{4} \not l-2 m_{f}\right)\{S(l)-S(l+k)\}\right]
$$


and we may redefine the loop momenta in the integrands to obtain

$$
\begin{aligned}
k^{\nu} T_{\mu \nu}^{(\phi f: \mathrm{a})}+k^{\nu} T_{\mu \nu}^{(\phi f: \mathrm{b})} & =3 i \omega k^{\nu} \int \frac{d^{4} l}{(2 \pi)^{4}} \operatorname{Tr}\left[\tilde{\gamma}_{\mu} S(l) \gamma_{\nu} S(l+p)\right] \\
& =-k^{\nu} T_{\mu \nu}^{(\phi f: \mathrm{c})}
\end{aligned}
$$

so that overall

$$
k^{\nu} T_{\mu \nu}^{(\phi f)}=0
$$

\subsubsection{W loop diagrams}

We begin by taking the expression for $k^{\nu} T_{\mu \nu}^{(\phi W: f)}$, using equation (3.20), and applying equation (6.9), obtaining

$$
k^{\nu} T_{\mu \nu}^{(\phi W: \mathrm{f})}=2 i M_{Z}^{2} \eta_{\mu \alpha} D_{Z}^{\alpha \beta}(k) \int \frac{d^{4} l}{(2 \pi)^{4}} N_{\gamma \delta \beta}(l,-l-k, k)\left[D_{W}^{\gamma \delta}(l+k)-D_{W}^{\gamma \delta}(l)\right] .
$$

We then redefine the loop momentum integration variable so that the integrand has a common coefficient $D_{W}^{\gamma \delta}(l)$, and apply equation (6.11), to obtain

$$
\begin{aligned}
k^{\nu} T_{\mu \nu}^{(\phi W: \mathrm{f})} & =2 i \omega M_{Z}^{2} \eta_{\mu \alpha} k^{\nu} R_{\gamma \delta \beta \nu} D_{Z}^{\alpha \beta}(k) \int \frac{d^{4} l}{(2 \pi)^{4}} D_{W}^{\gamma \delta}(l) \\
& =-k^{\nu} T_{\mu \nu}^{(\phi W: \mathrm{j})} .
\end{aligned}
$$

We also redefine the loop momenta integration variables in the expressions for $T_{\mu \nu}^{(\phi W: a)}$ and $T_{\mu \nu}^{(\phi W: \mathrm{b})}$ (equations (3.18) and (3.19), taking $l \rightarrow l-q$ and $l \rightarrow l+k$, respectively), and use equation (6.9), to write

$$
\begin{aligned}
k^{\nu} T_{\mu \nu}^{(\phi W: \mathrm{a})}+k^{\nu} T_{\mu \nu}^{(\phi W: \mathrm{b})}= & 2 i \omega M_{W}^{2} \eta_{\sigma \tau} \int \frac{d^{4} l}{(2 \pi)^{4}} N_{\alpha \beta \mu}(l-q,-l-k, p) D_{W}^{\alpha \tau}(l-q) \times \\
\times & \times\left[D_{W}^{\beta \sigma}(l+k)-D_{W}^{\beta \sigma}(l)\right]+ \\
& +2 i \omega M_{W}^{2} \eta_{\gamma \delta} \int \frac{d^{4} l}{(2 \pi)^{4}} N_{\alpha \beta \mu}(l,-l-p, p) D_{W}^{\beta \gamma}(l+p) \times \\
& \times\left[D_{W}^{\alpha \delta}(l+k)-D_{W}^{\alpha \delta}(l)\right] \\
= & 2 i \omega M_{W}^{2} \eta_{\sigma \tau} \int \frac{d^{4} l}{(2 \pi)^{4}} D_{W}^{\alpha \tau}(l)\left[N_{\alpha \beta \mu}(l-k,-l+k-p, p)-\right. \\
= & -2 i \omega M_{W}^{2} \eta_{\sigma \tau} k^{\nu} R_{\alpha \beta \mu \nu} \int \frac{d^{4} l}{(2 \pi)^{4}} D_{W}^{\alpha \tau}(l) D_{W}^{\beta \sigma}(l+q) \\
= & -k^{\nu} T_{\mu \nu}^{(\phi W: \mathrm{g})},
\end{aligned}
$$

where to obtain equation (6.39) we have used equation (6.11).

As the other diagrams evaluate to zero, we are now able to establish the result

$$
k^{\nu} T_{\mu \nu}^{(\phi W)}=0 .
$$




\subsubsection{Counterterm diagrams}

Contracting with $k^{\nu}$ the expressions for $F_{\mu \nu}^{(\phi \times: a)}$ and $F_{\mu \nu}^{(\phi \times: b)}$ given in equations (4.19) and (4.20) respectively, and recalling the expressions for $A(k)$ and $B$ given in equations (4.8) and (4.9) respectively, along with the expression for the propagator $D_{Z}^{\alpha \beta}(k)$ given in equation (2.25), it is straightforward to see that

$$
k^{\nu} F_{\mu \nu}^{(\phi \times: \mathrm{a})}+k^{\nu} F_{\mu \nu}^{(\phi \times: \mathrm{b})}=0 .
$$

Given that $F_{\mu \nu}^{(\phi \times: c)}=0$, we can therefore deduce that

$$
k^{\nu} F_{\mu \nu}^{(\phi \times)}=0 .
$$

\section{Calculation of the amplitude coefficients}

Having shown that the individual sets of diagrams (ones with fermion loops, ones with W boson loops, and ones with counterterms) separately satisfy the requisite conditions to give a contribution to the amplitudes matching the given general forms, we can now calculate these contributions separately. We begin by showing that the contribution to the amplitude from the counterterm diagrams is zero in both the decay involving a spin-2 graviton excitation and the decay involving a spin-0 graviton excitation. This will mean that the contributions from the other sets of diagrams must be finite, and so we should not be surprised when we see a "miraculous" cancellation of divergences in the case of decay to a spin-0 graviton excitation. Indeed, we note that as we have not made any particular assumptions about the number of fermions, we know that there cannot be a cancellation of infinities between the fermion loop diagrams and the $\mathrm{W}$ boson loop diagrams. This means that the contribution from each of the individual sets of diagrams must be finite.

\subsection{Counterterm diagrams contribution}

\subsubsection{Spin-2 case}

We note that there are no Levi-Civita tensors in any of the counterterm-containing diagrams that contribute to the decay amplitude in the spin-2 graviton excitation case (equations (4.16) to (4.18)). The diagrams therefore give no contribution to either $F_{1}^{(h)}$ or $F_{2}^{(h)}$, and we need only consider the contribution to $F^{(h)}$.

To consider the contribution to $F^{(h)}$ we need consider only one of the terms of which it is a coefficient, and (as in [1]) we choose the term $F^{(h)} k_{\lambda} k_{\rho} q_{\mu} q_{\nu}$ (noting that this term appears twice in the expression given in equation (5.20)). Equation (1.10) implies that

$$
\varepsilon_{\mathrm{Z}}^{\mu}(p) k_{\mu}=-\varepsilon_{\mathrm{Z}}^{\mu}(p) q_{\mu}
$$

so that terms requiring consideration also arise from terms of the form $k_{\lambda} k_{\rho} k_{\mu} q_{\nu}$.

For diagram (a), we note that the contribution has an overall coefficient of $k_{\nu}$, and therefore does not contribute to the amplitude. For diagram (b), we note that there is no term containing four momenta with Lorentz indices, so there is no contribution from this term either. Similarly for diagram (c), once we note that the term with a $B$ coefficient 
contains a factor of $p_{\mu}$ so provides no contribution to the amplitude, we can see that there is no remaining term with four Lorentz index-carrying momenta. The contribution to $F^{(h)}$ from the counterterm diagrams is therefore zero.

\subsubsection{Spin-0 case}

We note that, as in the spin-2 case, there are no Levi-Civita tensors in any of the countertermcontaining diagrams that contribute to the decay amplitude in the spin-0 graviton excitation case (equations (4.19) to 4.21) ). The diagrams therefore give no contribution to $F_{1}^{(\phi)}$, and we need only consider the contribution to $F^{(\phi)}$.

Similarly to the spin-2 case, we need consider only one of the terms of which $F^{(\phi)}$ is a coefficient, and we choose the term $k_{\mu} q_{\nu}$. In this case, the term is not repeated in the expression for the general form of the amplitude. Again, equation (1.10) implies that terms requiring consideration also arise from terms of the form $q_{\mu} q_{\nu}$.

It is sufficient for the counterterm diagrams to note that no diagram expression depends upon the momentum $q$ of the spin-0 particle, so that there is no contribution from the counterterms to the amplitude.

\subsection{Loop diagram contributions to the spin-2 amplitude coefficients}

We shall see that there are no Levi-Civita terms to be considered, so that the contributions to $F_{1}^{(h)}$ and $F_{2}^{(h)}$ are zero. In considering contributions to $F^{(h)}$, we shall again look at terms of the form $k_{\lambda} k_{\rho} q_{\mu} q_{\nu}\left(\right.$ or $\left.k_{\lambda} k_{\rho} k_{\mu} q_{\nu}\right)$.

\subsubsection{Fermion loop diagrams}

The determination of the contribution from diagrams containing fermion loops is very similar to the massless case considered in [1].

With respect to the contributions to $F_{1}^{(h)}$ and $F_{2}^{(h)}$, we note that for diagrams (c), (d), (e) and (f), any term with a Levi-Civita tensor also has a metric tensor symmetric in two of the indices of the Levi-Civita tensor, so that there is no contribution from any of these diagrams.

With respect to the contributions to $F^{(h)}$, we note that none of diagrams (c), (d), (e) and (f) contributes a term of the relevant form: this is straightforward to see for diagrams (c) and (d), which only depend upon $p$ and $k$ respectively; it is relatively easy to see for diagrams (e) and (f) if one uses the definitions of $c_{\lambda \rho \beta \nu}(p, k)$ and $c_{\lambda \rho \mu \alpha}^{Z}(p, k)$ given in equations (2.2) and (2.3), respectively.

It is therefore necessary only to consider contributions from diagrams (a) and (b). The contributions from diagrams (a) and (b) may be written in the form

$$
\begin{aligned}
& T_{\lambda \rho \mu \nu}^{(h f: \mathrm{a})}=i \int \frac{d^{4} l}{(2 \pi)^{4}} \frac{l_{\rho} \operatorname{Tr}\left[\tilde{\gamma}_{\mu}\left(l-\not k+m_{f}\right) \gamma_{\nu}\left(l+m_{f}\right) \gamma_{\lambda}\left(l+\not l+m_{f}\right)\right]}{\left[(l-k)^{2}-m_{f}^{2}\right]\left[(l+q)^{2}-m_{f}^{2}\right]\left(l^{2}-m_{f}^{2}\right)}, \\
& T_{\lambda \rho \mu \nu}^{(h f: \mathrm{b})}=i \int \frac{d^{4} l}{(2 \pi)^{4}} \frac{l_{\rho} \operatorname{Tr}\left[\tilde{\gamma}_{\mu}\left(l-\not l+m_{f}\right) \gamma_{\lambda}\left(l+m_{f}\right) \gamma_{\nu}\left(l+\not k+m_{f}\right)\right]}{\left[(l+k)^{2}-m_{f}^{2}\right]\left[(l-q)^{2}-m_{f}^{2}\right]\left(l^{2}-m_{f}^{2}\right)} .
\end{aligned}
$$


As in reference [1] we can use the cyclic property of the trace and charge conjugation relations

$$
C^{-1} \gamma^{\mu} C=-\gamma^{\mu T}, \quad C^{-1} \gamma^{\mu} \gamma^{5} C=\left(\gamma^{\mu} \gamma^{5}\right)^{T},
$$

to write the sum of these terms as

$$
T_{\lambda \rho \mu \nu}^{(h f: \mathrm{a})}+T_{\lambda \rho \mu \nu}^{(h f: \mathrm{b})}=2 i X_{f} \int \frac{d^{4} l}{(2 \pi)^{4}} \frac{f_{\lambda \rho \mu \nu}(l)}{\left[(l-k)^{2}-m_{f}^{2}\right]\left[(l+q)^{2}-m_{f}^{2}\right]\left(l^{2}-m_{f}^{2}\right)},
$$

where

$$
f_{\lambda \rho \mu \nu}(l)=l_{\rho} \operatorname{Tr}\left[\gamma_{\mu}\left(\not l-\not k+m_{f}\right) \gamma_{\nu}\left(\not l+m_{f}\right) \gamma_{\lambda}\left(\not l+\not l+m_{f}\right)\right] .
$$

As the trace does not contain a $\gamma_{5}$ term, the contribution to the amplitude will not contain a Levi-Civita tensor, and so the contributions to $F_{1}^{(h)}$ and $F_{2}^{(h)}$ are zero. A Feynman parameterization yields

$$
T_{\lambda \rho \mu \nu}^{(h f: \mathrm{a})}+T_{\lambda \rho \mu \nu}^{(h f: \mathrm{b})}=4 i X_{f} \int \frac{d^{4} l}{(2 \pi)^{4}} \int_{0}^{1} d x \int_{0}^{1-x} d y \frac{f_{\lambda \rho \mu \nu}(l+x k-y q)}{\left[l^{2}-m_{f}^{2}+y(1-x-y) m_{\vec{n}}^{2}+x y M_{Z}^{2}\right]^{3}},
$$

and considering only the terms we have previously noted, there are no divergences to be considered and we can integrate with respect to the loop momentum, obtaining a contribution to $F^{(h)}$ from each fermion in the theory of

$$
F^{(h f)}=-\frac{\kappa e g}{2 \pi^{2} \cos \theta_{W}} Q_{f} X_{f} J\left(m_{f}, m_{\vec{n}}, M_{Z}\right),
$$

where

$$
J(X, Y, Z)=\int_{0}^{1} d x \int_{0}^{1-x} d y \frac{x^{2} y(1-x-y)}{X^{2}-y(1-x-y) Y^{2}-x y Z^{2}} .
$$

\subsubsection{W loop diagrams}

The determination of contributions from diagrams containing $\mathrm{W}$ boson loops is also very similar to the massless case considered in [1].

There are no Levi-Civita terms in the individual diagram contributions, so the overall contributions to $F_{1}^{(h)}$ and $F_{2}^{(h)}$ are zero. We need therefore only consider the $k_{\lambda} k_{\rho} q_{\mu} q_{\nu}$-like terms to obtain a contribution to the coefficient $F^{(h)}$.

The contributions from diagrams (g) and (h) have no dependence on $k$, so cannot take the form we are considering, and therefore may be ignored. Similarly to the fermion case, the forms of $c_{\lambda \rho \alpha \nu}(p, k)$ and $c_{\lambda \rho \mu \alpha}^{Z}(p, k)$, given in equations (2.2) and (2.3), respectively, are such that the combinations $T_{\lambda \rho \mu \nu}^{(h W: \mathrm{e})}+T_{\lambda \rho \mu \nu}^{(h W: \mathrm{i})}$ and $T_{\lambda \rho \mu \nu}^{(h W: \mathrm{f})}+T_{\lambda \rho \mu \nu}^{(h W: \mathrm{j})}$ contain no relevant contributions to the coefficient $F^{(h)}$ (see [1] for more details). Expanding the expressions for diagrams (c) and (d), we see that no terms in the expressions contain a $q_{\nu}$ term, so these diagrams provide no relevant contribution. We are therefore left with only the contributions from diagrams (a) and (b). The contributions from these diagrams are equal: this may 
be seen by applying a change of variable in one of the integrands in the expressions for $T_{\lambda \rho \mu \nu}^{(h W: \mathrm{a})}$ and $T_{\lambda \rho \mu \nu}^{(h W: \mathrm{b})}$, or perhaps more straightforwardly by observing that the contributions obtained from the diagrams are independent of the direction in which the $\mathrm{W}$ boson loop is traversed.

It may be shown after some manipulation [1] that the contribution from diagram (b) is contained in the equation

$$
\begin{aligned}
T_{\lambda \rho \mu \nu}^{(h W: \mathrm{b})}= & 8 i k_{\lambda} k_{\rho} q_{\mu} q_{\nu}\left(6-\frac{M_{Z}^{2}}{M_{W}^{2}}\right) \times \\
& \times \int \frac{d^{4} l}{(2 \pi)^{4}} \int_{0}^{1} d x \int_{0}^{1-x} d y \frac{x^{2} y(1-x-y)}{\left[l^{2}-M_{W}^{2}+y(1-x-y) m_{\vec{n}}^{2}+x y M_{Z}^{2}\right]^{3}}+ \\
& + \text { non-contributing terms },
\end{aligned}
$$

and therefore, after performing the integration over the loop momentum, that the overall contribution from the $\mathrm{W}$ boson loop diagrams to the coefficient $F^{(h)}$ is

$$
F^{(h W)}=\frac{\kappa e g \cos \theta_{W}}{4 \pi^{2}}\left(6-\frac{M_{Z}^{2}}{M_{W}^{2}}\right) J\left(M_{W}, m_{\vec{n}}, M_{Z}\right),
$$

where $J(X, Y, Z)$ is defined in equation (7.9).

\subsection{Overall contribution to the spin-2 amplitude coefficients}

We have determined so far that the coefficients $F_{1}^{(h)}$ and $F_{2}^{(h)}$ are zero. We have determined also that the coefficient $F^{(h)}$ is given by

$$
\begin{aligned}
F^{(h)}= & \frac{\kappa e g}{4 \pi^{2} \cos \theta_{W}} \times \\
& \times\left[\cos ^{2} \theta_{W}\left(6-\frac{1}{\cos ^{2} \theta_{W}}\right) J\left(M_{W}, m_{\vec{n}}, M_{Z}\right)-2 \sum_{f} Q_{f} X_{f} J\left(m_{f}, m_{\vec{n}}, M_{Z}\right)\right] .
\end{aligned}
$$

To proceed, we approximate the analytically intractable integrals of the form $J(X, Y, Z)$. We note that all the integrals in which we are interested take the form $J\left(X, m_{\vec{n}}, M_{Z}\right)$, and since the mass of the Kaluza-Klein mode is constrained by $m_{\vec{n}} \leq M_{Z}$, we may consider the integral only in the cases where $0 \leq Y \leq Z$.

We consider the expression for $J(X, Y, Z)$ given in equation (7.9), and repeated here for convenience:

$$
J(X, Y, Z)=\int_{0}^{1} d x \int_{0}^{1-x} d y \frac{x^{2} y(1-x-y)}{X^{2}-y(1-x-y) Y^{2}-x y Z^{2}} .
$$

We note that over the range of integration, the maximum value of the expression $x y$ is $1 / 4$, and the maximum value of the expression $y(1-x-y)$ is also $1 / 4$. This allows us to consider two limiting cases for approximation of the integral: $X /(Y+Z) \gg 1 / 4$ and $X /(Y+Z) \ll 1 / 4$. With the constraint $0 \leq Y \leq Z$, it is sufficient to consider the cases $X / Z \gg 1 / 2$ and $X / Z \ll 1 / 4$. 
To evaluate the case $X / Z \gg 1 / 2$, we may approximate the integral by $J(X, 0,0)$, which is relatively straightforward to calculate, and yields the result

$$
J(X, 0,0)=\frac{1}{360 X^{2}} .
$$

To evaluate the case $X / Z \ll 1 / 2$, we may approximate the integral by $J(0, Y, Z)$. We may change the order of integration so as to perform the $x$ integral first; this is possible analytically. We obtain an integral polynomial in $(1-y)$ that we can evaluate analytically. Evaluating this integral, we obtain

$$
J(0, Y, Z)=\frac{1}{12\left(Z^{2}-Y^{2}\right)}-\frac{Z^{2}}{8\left(Z^{2}-Y^{2}\right)^{2}}+\frac{Y^{2} Z^{2}}{4\left(Z^{2}-Y^{2}\right)^{3}}+\frac{Y^{4} Z^{2}}{4\left(Z^{2}-Y^{2}\right)^{4}} \log \left(\frac{Y^{2}}{Z^{2}}\right) .
$$

To proceed, we use the identity

$$
\frac{B^{2}}{C^{2}-B^{2}} \equiv \frac{C^{2}}{C^{2}-B^{2}}-1
$$

to eliminate all terms with a $B^{2}$ numerator, as well as the identity

$$
\log \left(\frac{B^{2}}{C^{2}}\right)=\log \left(1-\frac{C^{2}-B^{2}}{C^{2}}\right)
$$

and expand the logarithm term (the expansion is valid for $0<Y \leq Z$, and the final answer turns out to be valid for $Y=0$ as well). We obtain

$$
J(0, Y, Z)=-\frac{1}{2} \sum_{j=1}^{\infty} \frac{1}{(j+1)(j+2)(j+3)} Z^{-2 j}\left(Z^{2}-Y^{2}\right)^{j-1} .
$$

We now return to our expression for $F^{(h)}$ given in equation (7.12). We approximate the integrals by using the form of equation (7.14) for $J\left(M_{W}, m_{\vec{n}}, M_{Z}\right)$ and $J\left(m_{t}, m_{\vec{n}}, M_{Z}\right)$, and the form of equation (7.18) for the integrals relating to fermions other than the top quark. This gives

$$
\begin{aligned}
F^{(h)}=\frac{\kappa e g}{4 \pi^{2} \cos \theta_{W} M_{Z}^{2}} & {\left[\frac{1}{360}\left(6-\frac{1}{\cos ^{2} \theta_{W}}\right)+\right.} \\
& +\left(5-\frac{40}{3} \sin ^{2} \theta_{W}\right)\left(\sum_{j=0}^{\infty} \frac{1}{(j+2)(j+3)(j+4)}\left(1-\frac{m_{\vec{n}}^{2}}{M_{Z}^{2}}\right)^{j}\right)- \\
& \left.-\frac{M_{Z}^{2}}{m_{t}^{2}}\left(\frac{1}{180}-\frac{2}{135} \sin ^{2} \theta_{W}\right)\right]
\end{aligned}
$$

(where we have used that $M_{W}=M_{Z} \cos \theta_{W}$ ). Using $e=g \sin \theta_{W}$ and $\sin ^{2} \theta_{W}=0.23$, along with a numerical value for $M_{Z} / m_{t}$ [25], we obtain the approximation

$$
F^{(h)}=\frac{\kappa e^{2}}{4 \pi^{2} M_{Z}^{2}}\left[0.030+4.6\left(\sum_{j=0}^{\infty} \frac{1}{(j+2)(j+3)(j+4)}\left(1-\frac{m_{\vec{n}}^{2}}{M_{Z}^{2}}\right)^{j}\right)\right] .
$$

This approximation agrees with that given in reference [1] in the case $m_{\vec{n}}=0$. 


\subsection{Loop diagram contributions to the spin-0 amplitude coefficients}

As in the spin-2 case, we shall see that there are no Levi-Civita terms to be considered, so that the contributions to $F_{1}^{(\phi)}$ are zero. In considering contributions to $F^{(\phi)}$, we shall again look at terms of the form $k_{\mu} q_{\nu}$.

\subsubsection{Fermion loop diagrams}

The contributions from diagrams (d) and (f) are zero, since the contributions only depend upon $k$, so they are not of a form to contribute to $F^{(\phi)}$ and symmetry considerations show that they do not contribute to $F_{1}^{(\phi)}$. A similar argument shows that diagram (c), which depends only upon $p$, does not contribute to either of the coefficients.

As diagram (e) trivially gives a zero contribution, it follows that it is necessary only to consider contributions from diagrams (a) and (b).

The methodology employed is very similar to that of the spin-2 case, developed in [1]. We begin by writing out expressions for the fermion propagators, so that we may write the contributions as

$$
\begin{aligned}
T_{\mu \nu}^{(\phi f: \mathrm{a})} & =-2 i \omega \int \frac{d^{4} l}{(2 \pi)^{4}} \frac{\operatorname{Tr}\left[\tilde{\gamma}_{\mu}\left(\not l-\not k+m_{f}\right) \gamma_{\nu}\left(\not l+m_{f}\right)\left(\frac{3}{2} \not+\frac{3}{4} \not l-2 m_{f}\right)\left(\not l+\not l+m_{f}\right)\right]}{\left[(l+q)^{2}-m_{f}^{2}\right]\left[(l-k)^{2}-m_{f}^{2}\right]\left[l^{2}-m_{f}^{2}\right]}, \\
T_{\mu \nu}^{(\phi f: \mathrm{b})} & =-2 i \omega \int \frac{d^{4} l}{(2 \pi)^{4}} \frac{\operatorname{Tr}\left[\tilde{\gamma}_{\mu}\left(\not-\not l+m_{f}\right)\left(\frac{3}{2} \not l-\frac{3}{4} \not l-2 m_{f}\right)\left(\not l+m_{f}\right) \gamma_{\nu}\left(\not l+\not k+m_{f}\right)\right]}{\left[(l+k)^{2}-m_{f}^{2}\right]\left[(l-q)^{2}-m_{f}^{2}\right]\left[l^{2}-m_{f}^{2}\right]} .
\end{aligned}
$$

For the second integral, we change the integration parameter to $-l$, and apply the cyclic property of the trace, the trace-reversal invariance of strings of gamma matrices, the invariance of transposing matrices inside the trace, in addition to the charge conjugation properties of the matrices. We are able to obtain a term in $Y_{f}$ that cancels with the corresponding term in the first integral (so that there is no Levi-Civita term and therefore no contribution to $F_{1}^{(\phi)}$ ), and a term in $X_{f}$ equal to the corresponding term in the first integral. We are therefore able to write

$$
T_{\mu \nu}^{(\phi f: \mathrm{a})}+T_{\mu \nu}^{(\phi f: \mathrm{b})}=-4 i \omega X_{f} \int \frac{d^{4} l}{(2 \pi)^{4}} \frac{f_{\mu \nu}(l)}{\left[(l-k)^{2}-m_{f}^{2}\right]\left[(l+q)^{2}-m_{f}^{2}\right]\left[l^{2}-m_{f}^{2}\right]}
$$

where

$$
f_{\mu \nu}(l)=\operatorname{Tr}\left[\gamma_{\mu}\left(\not l+\not l+m_{f}\right)\left(\frac{3}{2} l l+\frac{3}{4} \not l-2 m_{f}\right)\left(l+m_{f}\right) \gamma_{\nu}\left(\not-\not k+m_{f}\right)\right] .
$$


Applying a Feynman parameterization and continuing to $D$ dimensions, the equation becomes

$$
\begin{aligned}
T_{\mu \nu}^{(\phi f: \mathrm{a})}+T_{\mu \nu}^{(\phi f: \mathrm{b})}= & -8 i \omega X_{f} \mu^{(4-D)} \times \\
& \times \int \frac{d^{D} l}{(2 \pi)^{D}} \int_{0}^{1} d x \int_{0}^{1-x} d y \frac{f_{\mu \nu}(l+x k-y q)}{\left[l^{2}-m_{f}^{2}+y(1-x-y) m_{\vec{n}}^{2}+x y M_{Z}^{2}\right]^{3}} .
\end{aligned}
$$

We are interested in terms that will contribute to the coefficient of $k_{\mu} q_{\nu}$. We may use the symmetry properties of the $l$ integral to discard terms that are odd in $l$, and to replace

$$
\begin{aligned}
l_{\alpha} l_{\beta} & \rightarrow \frac{1}{D} \eta_{\alpha \beta} l^{2}, \\
l_{\alpha} l_{\beta} l_{\gamma} l_{\delta} & \rightarrow \frac{1}{D(D+2)}\left(\eta_{\alpha \beta} \eta_{\gamma \delta}+\eta_{\alpha \gamma} \eta_{\beta \delta}+\eta_{\alpha \delta} \eta_{\beta \gamma}\right)\left(l^{2}\right)^{2}
\end{aligned}
$$

(see for example page 477 of reference [26]). We find [24] that the term in the numerator of the integral containing $k_{\mu} q_{\nu}$ may be written as

$$
\begin{aligned}
k_{\mu} q_{\nu}[ & 3 M_{Z}^{2} x y\left(2-3 x-6 y+4 x y+4 y^{2}\right)+3 m_{\vec{n}}^{2} y(1-x-y)\left(1-3 x-4 y+4 x y+4 y^{2}\right)+ \\
& +m_{f}^{2}\left(5-3 x-20 y+20 x y+20 y^{2}\right)+ \\
& \left.+3 l^{2}\left(\left(-1+x+4 y-4 x y-4 y^{2}\right)+\frac{2}{D}\left(-1+3 x+8 y-8 x y-8 y^{2}\right)\right)\right] .
\end{aligned}
$$

We note that the integral appears to have a divergent term. However, it will turn out that the coefficient of the divergent term is zero after integration over the Feynman parameters.

It is possible, although not entirely straightforward, to evaluate the integral with the numerator given above. In order to do so, we adopt the following strategy:

- Perform the integrations over the loop momentum $l$. For the numerator terms not containing factors of $l$, we use the standard result. For the numerator terms containing factors of $l$, we use the results given in Appendix A. We obtain a result that appears to diverge as $D \rightarrow 4$.

- Order the Feynman parameter integration so as to perform the $x$ integral first.

- Perform the $x$ and $y$ integration for the coefficients of the terms that appear to be $D \rightarrow 4$ divergent. (These are also the coefficients of $\gamma$ and $\log (4 \pi)$ " $\overline{\mathrm{MS}}$-like" terms, which we therefore consider at the same time.) The terms vanish.

- To simplify the algebra, rewrite the numerator of the $1 /\left[m_{f}^{2}-y(1-x-y) m_{\vec{n}}^{2}-x y M_{Z}^{2}\right]$ term to eliminate terms with numerator coefficients involving $m_{f}^{2}$. (Note that doing this before approximating the integral in the way done below makes no difference when making the light fermion approximation given, and is in fact equivalent to including an extra term in the series expansion for the fraction when making the heavy fermion approximation given.) 
- At this stage, there are terms in the integrand that do not contain masses (these arise from the step above, and from the "anomalous" terms arising from the loop integration that looks like equation (A.1)). Integrate these terms with respect to the Feynman parameters.

- Integrate the logarithmic term with respect to $x$ by parts, obtaining a contribution to the $1 /\left[m_{f}^{2}-y(1-x-y) m_{\vec{n}}^{2}-x y M_{Z}^{2}\right]$ term and a logarithmic term to be integrated with respect to $y$ only.

- Integrate the remaining logarithmic term with respect to $y$ by parts. One of the resultant terms is zero. Approximate the denominator of the other term by considering the cases $m_{f}^{2} \ll M_{Z}^{2} / 4$ (in which case take $m_{f}^{2}=0$ ) and $m_{f}^{2} \gg M_{Z}^{2} / 4$ (in which case take $\left.M_{Z}^{2}=0\right)$.

- In order to perform the remaining integral (the integrand has a denominator of $\left.\left[m_{f}^{2}-y(1-x-y) m_{\vec{n}}^{2}-x y M_{Z}^{2}\right]\right)$, approximate the denominator of the integrand by considering the cases $m_{f}^{2} \ll M_{Z}^{2} / 4$ (in which case take $m_{f}^{2}=0$ ) and $m_{f}^{2} \gg M_{Z}^{2} / 2$ (in which case take $\left.M_{Z}^{2}=0, m_{\vec{n}}^{2}=0\right)$. The latter approximation leads to a relatively straightforward integral. For the former approximation, the integral may be evaluated by considering the numerator and denominator as polynomials in $x$, and writing the integrand in quotient+remainder form.

Following this strategy, we obtain that for each fermion, the contribution to the amplitude coefficient $F^{(\phi)}$ is

$$
F^{(\phi f)}= \begin{cases}0 & \text { for } m_{f}^{2} \ll \frac{1}{4} M_{Z}^{2}, \\ -\frac{\omega X_{f}}{(4 \pi)^{2}}\left[\frac{8}{3}+\frac{1}{45} \frac{7 m_{\vec{n}}^{2}+11 M_{Z}^{2}}{m_{f}^{2}}\right] & \text { for } m_{f}^{2} \gg \frac{1}{2} M_{Z}^{2} .\end{cases}
$$

\subsubsection{W loop diagrams}

There are no Levi-Civita terms in the individual diagram contributions, so the overall contribution to $F_{1}^{(\phi)}$ is zero. We need therefore only consider the $k_{\mu} q_{\nu}$-like terms to obtain a contribution to the coefficient $F^{(\phi)}$.

The contributions from diagrams (f) and ( $\mathrm{j}$ ) are zero, since the contributions only depend upon $k$, and therefore do not yield a term of the form we are considering.

With the exception of diagrams (a), (b) and (g), the other diagrams trivially give a zero contribution. We need to consider the contributions from each of diagrams (a), (b) and $(\mathrm{g})$.

We begin by considering the contributions from diagrams (a) and (b). These diagrams yield an identical contribution. We may see this through consideration of the integral that contributes to $T_{\mu \nu}^{(\phi W: \mathrm{b})}$, given in equation (3.19). If we take this integral, change the integration parameter taking $l \rightarrow-l-q$, relabel the dummy suffices and note that

$$
D_{W}^{\alpha \beta}(l)=D_{W}^{\alpha \beta}(-l)
$$


and

$$
N_{\alpha \beta \gamma}\left(k_{1}, k_{2}, k_{3}\right)=-N_{\beta \alpha \gamma}\left(k_{2}, k_{1}, k_{3}\right),
$$

we are able to obtain the expression for $T_{\mu \nu}^{(\phi W: a)}$. We can therefore obtain the contribution from both diagrams by considering the form of the contribution from one of the diagrams, and we choose diagram (b). We write the contribution in the form

$$
T_{\mu \nu}^{(\phi W: \mathrm{a})}+T_{\mu \nu}^{(\phi W: \mathrm{b})}=\frac{4 i \omega}{M_{W}^{2}} \int \frac{d^{4} l}{(2 \pi)^{4}} \frac{S_{\mu \nu}(l)}{\left[(l-k)^{2}-M_{W}^{2}\right]\left[(l+q)^{2}-M_{W}^{2}\right]\left[l^{2}-M_{W}^{2}\right]},
$$

where

$$
\begin{aligned}
S_{\mu \nu}(l)= & M_{W}^{4} \eta_{\gamma \delta} N_{\alpha \beta \mu}(l-k,-l-q, p) N_{\sigma \tau \nu}(l,-l+k,-k) \times \\
& \times\left(-\eta^{\alpha \tau}+\frac{(l-k)^{\alpha}(l-k)^{\tau}}{M_{W}^{2}}\right)\left(-\eta^{\delta \sigma}+\frac{l^{\delta} l^{\sigma}}{M_{W}^{2}}\right)\left(-\eta^{\beta \gamma}+\frac{(l+q)^{\beta}(l+q)^{\gamma}}{M_{W}^{2}}\right) .
\end{aligned}
$$

Applying a Feynman parameterization and continuing to $D$ dimensions, the equation becomes

$$
\begin{aligned}
T_{\mu \nu}^{(\phi W: \mathrm{a})}+T_{\mu \nu}^{(\phi W: \mathrm{b})}= & \frac{8 i \omega}{M_{W}^{2}} \mu^{(4-D)} \times \\
& \times \int \frac{d^{D} l}{(2 \pi)^{D}} \int_{0}^{1} d x \int_{0}^{1-x} d y \frac{S_{\mu \nu}(l+x k-y q)}{\left[l^{2}-M_{W}^{2}+y(1-x-y) m_{\vec{n}}^{2}+x y M_{Z}^{2}\right]^{3}} .
\end{aligned}
$$

We leave the equation in this form for now, and turn to the contribution from diagram (g). Unlike the corresponding diagram for spin-2 particle decay, this diagram provides a contribution to the amplitude coefficient. We write this contribution in the form

$$
T_{\mu \nu}^{(\phi W: \mathrm{g})}=\frac{2 i \omega}{M_{W}^{2}} \frac{U_{\mu \nu}(l)}{\left[l^{2}-M_{W}^{2}\right]\left[(l+q)^{2}-M_{W}^{2}\right]},
$$

where

$$
U_{\mu \nu}(l)=M_{W}^{4} \eta_{\sigma \tau} R_{\alpha \beta \mu \nu}\left(-\eta^{\tau \alpha}+\frac{l^{\tau} l^{\alpha}}{M_{W}^{2}}\right)\left(-\eta^{\beta \sigma}+\frac{(l+q)^{\beta}(l+q)^{\sigma}}{M_{W}^{2}}\right) .
$$

We wish to combine this contribution with that for diagrams (a) and (b), so we write it in the form

$$
T_{\mu \nu}^{(\phi W: \mathrm{g})}=\frac{2 i \omega}{M_{W}^{2}} \int \frac{d^{4} l}{(2 \pi)^{4}} \frac{U_{\mu \nu}(l)\left[(l-k)^{2}-M_{W}^{2}\right]}{\left[l^{2}-M_{W}^{2}\right]\left[(l+q)^{2}-M_{W}^{2}\right]\left[(l-k)^{2}-M_{W}^{2}\right]} .
$$

Applying a Feynman parameterization and continuing to $D$ dimensions, the equation becomes

$$
\begin{aligned}
T_{\mu \nu}^{(\phi W: \mathrm{g})}= & \frac{4 i \omega}{M_{W}^{2}} \mu^{(4-D)} \times \\
& \times \int \frac{d^{D} l}{(2 \pi)^{D}} \int_{0}^{1} d x \int_{0}^{1-x} d y \frac{U_{\mu \nu}(l+x k-y q)\left[(l+(x-1) k-y q)^{2}-M_{W}^{2}\right]}{\left[l^{2}-M_{W}^{2}+y(1-x-y) m_{\vec{n}}^{2}+x y M_{Z}^{2}\right]^{3}} .
\end{aligned}
$$


We may now combine the contributions from diagrams (a), (b) and (g). Using the symmetry properties of the $l$ integral given in equations (7.26) and (7.27), and taking a factor of $4 i \omega / M_{W}^{2}$ outside the integral, we find [24] that the term in the numerator of the integrand containing $k_{\mu} q_{\nu}$ may be written as

$$
\begin{aligned}
k_{\mu} q_{\nu}[ & M_{Z}^{4}\left(2 x y^{2}-2 x y^{3}-\frac{1}{2} x^{2} y-x^{2} y^{2}+\frac{1}{2} x^{3} y-x^{3} y^{2}\right)+ \\
& +m_{\vec{n}}^{2} M_{Z}^{2}\left(2 y^{2}-4 y^{3}+2 y^{4}-3 x y^{2}+3 x y^{3}+x^{2} y-\right. \\
& \left.\quad-\frac{3}{2} x^{2} y^{2}+2 x^{2} y^{3}-x^{3} y+2 x^{3} y^{2}\right)+ \\
+ & m_{\vec{n}}^{4}\left(-x y^{2}+2 x y^{3}-x y^{4}-\frac{1}{2} x^{2} y+\frac{5}{2} x^{2} y^{2}-2 x^{2} y^{3}+\frac{1}{2} x^{3} y-x^{3} y^{2}\right)+ \\
+ & M_{W}^{2} M_{Z}^{2}\left(-2+4 y-6 y^{2}-2 x+2 x y+\frac{1}{2} x^{2}-5 x^{2} y\right)+ \\
+ & M_{W}^{2} m_{\vec{n}}^{2}\left(-5 x y+5 x y^{2}-\frac{1}{2} x^{2}+5 x^{2} y\right)+ \\
+ & M_{W}^{4}\left(12+8 y-8 y^{2}-4 x-8 x y+D\left(-8 y+8 y^{2}+8 x y\right)\right)+ \\
+ & l^{2}\left\{M _ { Z } ^ { 2 } \left(\left(-2+2 y^{2}+2 x+x y-\frac{1}{2} x^{2}+2 x^{2} y\right)+\right.\right. \\
& \left.+\left(l^{2}\right)^{2}\left\{-x+\frac{2}{D}(1-2 x)\right\}\right] \\
& \left.+\frac{2}{D}\left(2-4 y+4 y^{2}+x+x y-x^{2}+4 x^{2} y\right)\right)+ \\
& +m_{\vec{n}}^{2}\left(\left(2 x y-2 x y^{2}+\frac{1}{2} x^{2}-2 x^{2} y\right)+\right. \\
& \left.\left.+(4+5 x)+\frac{2}{D}(-12+5 x)\right)\right\}
\end{aligned}
$$

We evaluate this integral using a similar strategy to that for the fermion loop case. To simplify the algebra, we rewrite the numerators of some fractional terms in order to obtain fewer terms requiring the application of approximations. Subdominant terms will be affected by the order in which rewriting a numerator and approximating the denominator is performed. However, this effect will be negligible at the order of the approximations made to the fractions themselves. Nevertheless, we record the order in which we proceed through the algebra, so that it is possible to reproduce the result.

The strategy adopted is as follows:

- Perform the integrations over the loop momentum $l$. As in the fermion case, for the 
numerator terms not containing factors of $l$, we use the standard result, and for the numerator terms containing factors of $l$, we use the results given in Appendix A. Again we obtain a result that, at first, appears to diverge as $D \rightarrow 4$.

- Order the Feynman parameter integration so as to perform the $x$ integral first.

- Perform the $x$ and $y$ integration for the coefficients of the terms that appear to be $D \rightarrow 4$ divergent (and of the terms that are " $\overline{M S}$-like"). The terms vanish.

- Rewrite the numerator of the $1 /\left[M_{W}^{2}-y(1-x-y) m_{\vec{n}}^{2}-x y M_{Z}^{2}\right]$ term to eliminate terms with numerator coefficients involving $M_{W}^{2}$ (as in the fermionic case, when taken with the approximation for the denominator below this is equivalent to adding higher order corrections in the series expansion for the fraction).

- Integrate the terms that are non-fractional and do not contain a logarithm.

- Integrate the logarithmic term with respect to $x$ by parts, obtaining a contribution to the $1 /\left[M_{W}^{2}-y(1-x-y) m_{\vec{n}}^{2}-x y M_{Z}^{2}\right]$ term and a logarithmic term to be integrated with respect to $y$ only. Rewrite the numerator of the fraction to eliminate terms with coefficients involving $M_{W}^{2}$.

- Integrate the remaining logarithmic term with respect to $y$ by parts. The only term that results is an integral with fractional integrand. This term can be integrated if we approximate the denominator by $M_{W}^{2}$. (Note that the result below does not involve rewriting the numerator term with $M_{W}^{2}$ coefficient for this term only.)

- Perform the remaining double integral by approximating the denominator of the fractional integrand by $M_{W}^{2}$.

Following this strategy, we find that the contribution from the $\mathrm{W}$ boson loop diagrams to the coefficient $F^{(\phi)}$ is

$$
F^{(\phi W)}=\kappa e g \cos \theta_{W} \frac{\omega}{(4 \pi)^{2}}\left[14+\frac{11}{15} \frac{m_{\vec{n}}^{2}}{M_{W}^{2}}-\frac{37}{15} \frac{M_{Z}^{2}}{M_{W}^{2}}+\frac{19}{210} \frac{m_{\vec{n}}^{4}}{M_{W}^{4}}+\frac{1}{210} \frac{m_{\vec{n}}^{2} M_{Z}^{2}}{M_{W}^{4}}-\frac{3}{20} \frac{M_{Z}^{4}}{M_{W}^{4}}\right]
$$

\subsection{Overall contribution to the spin-0 amplitude coefficients}

We have determined so far that the coefficient $F_{1}^{(\phi)}$ is zero. We have also determined that the coefficient $F^{(\phi)}$ is given by

$$
\begin{aligned}
F^{(\phi)}= & \kappa e g \cos \theta_{W} \frac{\omega}{(4 \pi)^{2}}\left[14+\frac{11}{15} \frac{m_{\vec{n}}^{2}}{M_{W}^{2}}-\frac{37}{15} \frac{M_{Z}^{2}}{M_{W}^{2}}+\frac{19}{210} \frac{m_{\vec{n}}^{4}}{M_{W}^{4}}+\frac{1}{210} \frac{m_{\vec{n}}^{2} M_{Z}^{2}}{M_{W}^{4}}-\frac{3}{20} \frac{M_{Z}^{4}}{M_{W}^{4}}\right]- \\
& -\frac{\kappa e Q_{t} g}{2 \cos \theta_{W}} \frac{\omega X_{t}}{(4 \pi)^{2}}\left[\frac{8}{3}+\frac{1}{45} \frac{7 m_{\vec{n}}^{2}+11 M_{Z}^{2}}{m_{t}^{2}}\right] .
\end{aligned}
$$


Substituting a value for $Q_{t} X_{t}$ (and including the colour degrees of freedom), this becomes

$$
\begin{aligned}
F^{(\phi)}= & \kappa e g \cos \theta_{W} \frac{\omega}{(4 \pi)^{2}}\left[14+\frac{11}{15} \frac{m_{\vec{n}}^{2}}{M_{W}^{2}}-\frac{37}{15} \frac{M_{Z}^{2}}{M_{W}^{2}}+\frac{19}{210} \frac{m_{\vec{n}}^{4}}{M_{W}^{4}}+\frac{1}{210} \frac{m_{\vec{n}}^{2} M_{Z}^{2}}{M_{W}^{4}}-\frac{3}{20} \frac{M_{Z}^{4}}{M_{W}^{4}}\right]- \\
& -\frac{\kappa e g}{2 \cos \theta_{W}} \frac{\omega}{(4 \pi)^{2}}\left(1-\frac{8}{3} \sin ^{2} \theta_{W}\right)\left[\frac{8}{3}+\frac{1}{45} \frac{7 m_{\vec{n}}^{2}+11 M_{Z}^{2}}{m_{t}^{2}}\right],
\end{aligned}
$$

and using $M_{W}=M_{Z} \cos \theta_{W}, e=g \sin \theta_{W}$ and $\sin ^{2} \theta_{W}=0.23$, along with a numerical value for $M_{Z} / m_{t}$ [25], we obtain the approximation

$$
F^{(\phi)}=\frac{\kappa e^{2} \omega}{(4 \pi)^{2}}\left[18+1.7 \frac{m_{\vec{n}}^{2}}{M_{Z}^{2}}+0.28 \frac{m_{\vec{n}}^{4}}{M_{Z}^{4}}\right] .
$$

\section{Calculation of the decay width}

\subsection{Decays involving spin-2 Kaluza-Klein particles}

For the decay to a spin-2 KK excitation, the matrix element is given by

$$
\mathcal{M}^{(h)}=\mathcal{E}^{\lambda \rho *}(q) \varepsilon^{\nu *}(k) \varepsilon_{\mathrm{Z}}^{\mu}(p) F^{(h)}\left[\left(k_{\lambda} q_{\nu}-k \cdot q \eta_{\nu \lambda}\right)\left(k_{\rho} q_{\mu}-k \cdot q \eta_{\mu \rho}\right)+(\lambda \leftrightarrow \rho)\right] .
$$

To obtain the square of the matrix element, we need the polarisation sum formulae

$$
\begin{aligned}
& \sum_{\mathrm{pol}} \mathcal{E}^{\lambda \rho *}(q) \mathcal{E}^{\lambda^{\prime} \rho^{\prime}}(q)=\frac{1}{2}[\left(\eta^{\lambda \lambda^{\prime}}-\frac{q^{\lambda} q^{\lambda^{\prime}}}{m_{\vec{n}}^{2}}\right)\left(\eta^{\rho \rho^{\prime}}-\frac{q^{\rho} q^{\rho^{\prime}}}{m_{\vec{n}}^{2}}\right)+ \\
&+\left(\eta^{\lambda \rho^{\prime}}-\frac{q^{\lambda} q^{\rho^{\prime}}}{m_{\vec{n}}^{2}}\right)\left(\eta^{\lambda^{\prime} \rho}-\frac{q^{\lambda^{\prime}} q^{\rho}}{m_{\vec{n}}^{2}}\right)- \\
&\left.-\frac{2}{3}\left(\eta^{\lambda \rho}-\frac{q^{\lambda} q^{\rho}}{m_{\vec{n}}^{2}}\right)\left(\eta^{\lambda^{\prime} \rho^{\prime}}-\frac{q^{\lambda^{\prime}} q^{\rho^{\prime}}}{m_{\vec{n}}^{2}}\right)\right] \\
& \sum_{\mathrm{pol}} \varepsilon^{\nu *}(k) \varepsilon^{\nu^{\prime}}(k)=-\eta^{\nu \nu^{\prime}}, \\
& \sum_{\mathrm{pol}} \varepsilon_{\mathrm{Z}}^{\mu}(p) \varepsilon_{Z}^{\mu^{\prime} *}(p)=-\eta^{\mu \mu^{\prime}}+\frac{p^{\mu} p^{\mu^{\prime}}}{M_{Z}^{2}}
\end{aligned}
$$

(we note that the formula for the gravitational polarisation sum [7, 22] differs from the massless case presented in [1]). This gives [24]

$$
\left|\mathcal{M}^{(h)}\right|^{2}=\left|F^{(h)}\right|^{2}\left(\frac{\left(M_{Z}^{2}-m_{\vec{n}}^{2}\right)^{4}\left(7 M_{Z}^{2}+3 m_{\vec{n}}^{2}\right)}{36 M_{Z}^{2}}\right) .
$$

We note that as $m_{\vec{n}} \rightarrow 0$, we recover the expression obtained for the case of decay to a massless graviton [1], save that using the massive gravitational polarisation spin sum causes a difference by a factor of $7 / 2$ from using the massless gravitational polarisation spin sum.

The decay width $\Gamma^{(h, \vec{n})}$ to a single spin-2 KK mode is given by

$$
\Gamma^{(h, \vec{n})}=\frac{p^{*}}{32 \pi^{2} M_{Z}^{2}} \int\left|\mathcal{M}^{(h)}\right|^{2} d \Omega,
$$


where

$$
p^{*}=\frac{1}{2 M_{Z}}\left(M_{Z}^{2}-m_{\vec{n}}^{2}\right)
$$

This gives

$$
\Gamma^{(h, \vec{n})}=\frac{1}{576 \pi M_{Z}^{5}}\left(M_{Z}^{2}-m_{\vec{n}}^{2}\right)^{5}\left(7 M_{Z}^{2}+3 m_{\vec{n}}^{2}\right)\left|F^{(h)}\right|^{2}
$$

Substituting the expression for $F^{(h)}$ obtained in equation $(\overline{7.20})$, and recalling that $\alpha=$ $e^{2} / 4 \pi$ and $\kappa=\sqrt{8 \pi G}$, we obtain

$$
\begin{aligned}
\Gamma^{(h, \vec{n})}= & \frac{\alpha^{2} G M_{Z}}{72 \pi^{2}}\left(1-\frac{m_{\vec{n}}^{2}}{M_{Z}^{2}}\right)^{5}\left(7 M_{Z}^{2}+3 m_{\vec{n}}^{2}\right) \times \\
\times & {\left[0.00088+0.27\left(\sum_{j=0}^{\infty} \frac{1}{(j+2)(j+3)(j+4)}\left(1-\frac{m_{\vec{n}}^{2}}{M_{Z}^{2}}\right)^{j}\right)+\right.} \\
& \left.+21\left(\sum_{i=0}^{\infty} \sum_{j=0}^{\infty} \frac{1}{(i+2)(i+3)(i+4)(j+2)(j+3)(j+4)}\left(1-\frac{m_{\vec{n}}^{2}}{M_{Z}^{2}}\right)^{i+j}\right)\right] .
\end{aligned}
$$

To obtain the total decay width involving spin-2 graviton excitations, we must sum over the excitation levels. Following [7], we make a continuum approximation for the density of states at a given mass level. Given a common compactification radius $R / 2 \pi$, the density of states over which we should integrate is

$$
\rho\left(m_{\vec{n}}\right)=\frac{R^{n} m_{\vec{n}}^{n-2}}{(4 \pi)^{n / 2} \Gamma(n / 2)},
$$

that is, the derivative with respect to $m_{\vec{n}}^{2}$ of the volume of a $n$-dimensional hypersphere of radius $r=\sqrt{\vec{n}^{2}}$. That is, the total decay width is approximated by

$$
\Gamma_{\text {tot }}^{(h)}=\int_{0}^{M_{Z}^{2}} d m_{\vec{n}}^{2} \Gamma^{(h, \vec{n})} \rho\left(m_{\vec{n}}\right) .
$$

We change the integration variable to $m_{\vec{n}}^{2} / M_{Z}^{2}$ and expand the resultant beta functions in 
terms of gamma functions, obtaining

$$
\begin{aligned}
\Gamma_{\text {tot }}^{(h)}= & \frac{\alpha^{2} G M_{Z}^{3+n} R^{n}}{72 \pi^{2}(4 \pi)^{n / 2}} \times \\
\times & {\left[0.00088\left(\frac{7 \cdot 5 !}{\Gamma\left(\frac{n}{2}+6\right)}+\frac{3 \cdot \frac{n}{2} \cdot 5 !}{\Gamma\left(\frac{n}{2}+7\right)}\right)+\right.} \\
& +0.27\left\{\sum_{j=0}^{\infty} \frac{1}{(j+2)(j+3)(j+4)}\left(\frac{7 \cdot(j+5) !}{\Gamma\left(\frac{n}{2}+j+6\right)}+\frac{3 \cdot \frac{n}{2} \cdot(j+5) !}{\Gamma\left(\frac{n}{2}+j+7\right)}\right)\right\}+ \\
& +21\left\{\sum_{i=0}^{\infty} \sum_{j=0}^{\infty} \frac{1}{(i+2)(i+3)(i+4)(j+2)(j+3)(j+4)} \times\right. \\
& \left.\left.\times\left(\frac{7 \cdot(i+j+5) !}{\Gamma\left(\frac{n}{2}+i+j+6\right)}+\frac{3 \cdot \frac{n}{2} \cdot(i+j+5) !}{\Gamma\left(\frac{n}{2}+i+j+7\right)}\right)\right\}\right]
\end{aligned}
$$

We note that the width tends to zero as $n \rightarrow \infty$, showing that as the number of dimensions increases for large $n$, the phase space increase dominates the increase in the number of $\mathrm{KK}$ states to reduce the decay width.

\subsection{Decays involving spin-0 Kaluza-Klein particles}

For the decay to a spin-0 KK excitation, the matrix element is given by

$$
\mathcal{M}^{(\phi)}=\varepsilon^{\nu *}(k) \varepsilon_{\mathrm{Z}}^{\mu}(p) F^{(\phi)}\left(k_{\mu} q_{\nu}-k \cdot q \eta_{\mu \nu}\right)
$$

Using equations (8.3) and (8.4), we obtain 24]

$$
\left|\mathcal{M}^{(\phi)}\right|^{2}=\frac{1}{6}\left(M_{Z}^{2}-m_{\vec{n}}^{2}\right)^{2}\left|F^{(\phi)}\right|^{2} .
$$

The decay width $\Gamma^{(\phi, \vec{n})}$ to a single spin-0 KK mode is given by

$$
\Gamma^{(\phi, \vec{n})}=\frac{p^{*}}{32 \pi^{2} M_{Z}^{2}} \int\left|\mathcal{M}^{(\phi)}\right|^{2} d \Omega
$$

where, as before,

$$
p^{*}=\frac{1}{2 M_{Z}}\left(M_{Z}^{2}-m_{\vec{n}}^{2}\right) .
$$

This gives

$$
\Gamma^{(\phi, \vec{n})}=\frac{1}{96 \pi M_{Z}^{3}}\left(M_{Z}^{2}-m_{\vec{n}}^{2}\right)^{3}\left|F^{(\phi)}\right|^{2} .
$$


Substituting the expression for $F^{(\phi)}$ obtained in equation (7.43), again using that $\alpha=e^{2} / 4 \pi$ and $\kappa=\sqrt{8 \pi G}$, and recalling that $\omega^{2}=2 /(3(n+2))$, we obtain

$$
\begin{aligned}
\Gamma^{(\phi, \vec{n})}= & \frac{\alpha^{2} G}{288 \pi^{2}(n+2) M_{Z}^{3}}\left(M_{Z}^{2}-m_{\vec{n}}^{2}\right)^{3} \times \\
& \times\left[330+63 \frac{m_{\overrightarrow{\vec{n}}}^{2}}{M_{Z}^{2}}+13 \frac{m_{\vec{n}}^{4}}{M_{Z}^{4}}+0.97 \frac{m_{\vec{n}}^{6}}{M_{Z}^{6}}+0.078 \frac{m_{\vec{n}}^{8}}{M_{Z}^{8}}\right] .
\end{aligned}
$$

To obtain the total decay width involving spin-0 graviton excitations, we again approximate by an integral the sum over the excitation levels. Recalling that we have $(n-1)$ spin-0 particles at each mass level, the total decay width is approximated by

$$
\Gamma_{\text {tot }}^{(\phi)}=\int_{0}^{M_{Z}^{2}} d m_{\vec{n}}^{2} \Gamma^{(\phi, \vec{n})}(n-1) \rho\left(m_{\vec{n}}\right)
$$

As in the spin-2 case, we change the integration variable to $m_{\vec{n}}^{2} / M_{Z}^{2}$ and expand the resultant beta functions in terms of gamma functions, obtaining

$$
\begin{aligned}
\Gamma_{\text {tot }}^{(\phi)}= & \frac{\alpha^{2} G M_{Z}^{3+n} R^{n}}{48 \pi^{2}(4 \pi)^{n / 2}} \frac{(n-1)}{(n+2)} \times \\
& \times\left[\frac{330}{\Gamma\left(\frac{n}{2}+4\right)}-\frac{63 \cdot \frac{n}{2}}{\Gamma\left(\frac{n}{2}+5\right)}+\frac{13 \cdot \frac{n}{2} \cdot\left(\frac{n}{2}+1\right)}{\Gamma\left(\frac{n}{2}+6\right)}-\frac{0.97 \cdot \frac{n}{2} \cdot\left(\frac{n}{2}+1\right) \cdot\left(\frac{n}{2}+2\right)}{\Gamma\left(\frac{n}{2}+7\right)}-\right. \\
& \left.\quad-\frac{0.078 \cdot \frac{n}{2} \cdot\left(\frac{n}{2}+1\right) \cdot\left(\frac{n}{2}+2\right) \cdot\left(\frac{n}{2}+3\right)}{\Gamma\left(\frac{n}{2}+8\right)}\right] .
\end{aligned}
$$

As in the spin-2 case, the decay width tends to zero as $n \rightarrow \infty$. We note that the width is zero for $n=1$, which we should expect as there are no spin- 0 excited states in the case of one extra dimension. We note also that whilst the width should be zero for $n=0$, the expression above does not give zero for $n=0$. However, the extra-dimensional spin sum identities of equation (1.5) are only valid for $n>0$.

\subsection{Overall decay width to photon and Kaluza-Klein graviton excitation}

We may now use equations (8.12) and 8.20 to write down an approximation for the full decay width at one loop of the $\mathrm{Z}$ boson to a photon and a Kaluza-Klein excitation in the 
ADD scenario:

$$
\begin{aligned}
& \Gamma_{\text {tot }}=\frac{\alpha^{2} G M_{Z}^{3+n} R^{n}}{72 \pi^{2}(4 \pi)^{n / 2}} \times \\
& \times\left[0.00088\left(\frac{7 \cdot 5 !}{\Gamma\left(\frac{n}{2}+6\right)}+\frac{3 \cdot \frac{n}{2} \cdot 5 !}{\Gamma\left(\frac{n}{2}+7\right)}\right)+\right. \\
&+0.27\left\{\sum_{j=0}^{\infty} \frac{1}{(j+2)(j+3)(j+4)}\left(\frac{7 \cdot(j+5) !}{\Gamma\left(\frac{n}{2}+j+6\right)}+\frac{3 \cdot \frac{n}{2} \cdot(j+5) !}{\Gamma\left(\frac{n}{2}+j+7\right)}\right)\right\}+ \\
&+21\left\{\sum_{i=0}^{\infty} \sum_{j=0}^{\infty} \frac{1}{(i+2)(i+3)(i+4)(j+2)(j+3)(j+4)} \times\right. \\
&+\frac{3}{2} \frac{(n-1)}{(n+2)}\left\{\frac{330}{\Gamma\left(\frac{n}{2}+4\right)}-\frac{63 \cdot \frac{n}{2}}{\Gamma\left(\frac{n}{2}+5\right)}+\frac{13 \cdot \frac{n}{2} \cdot\left(\frac{n}{2}+1\right)}{\Gamma\left(\frac{n}{2}+6\right)}-\right. \\
&\left.\left.\quad-\frac{0.97 \cdot \frac{n}{2} \cdot\left(\frac{n}{2}+1\right) \cdot\left(\frac{n}{2}+2\right)}{\Gamma\left(\frac{n}{2}+7\right)}-\frac{0.078 \cdot \frac{n}{2} \cdot\left(\frac{n}{2}+1\right) \cdot\left(\frac{n}{2}+2\right) \cdot\left(\frac{n}{2}+3\right)}{\Gamma\left(\frac{n}{2}+8\right)}\right\}\right]
\end{aligned}
$$

Two comments about this expression are inherited from the constituent expressions of equations (8.12) and $(8.20)$. Firstly, the overall expression does not reduce to the case of a single, massless graviton for $n=0$, because the extra-dimensional spin sum formula in equation (1.5) is only valid for $n \geq 1$. Secondly, the overall width tends to zero as $n \rightarrow \infty$, so that this process becomes less distinguishable from a Standard Model background as the number of extra dimensions increases.

Tables 1 and 2 give numerical values ${ }^{3}$ for the contributions inside the square brackets in equation (8.21). It is notable that the contributions from the decays to spin-0 excitations are dominant.

In order to estimate the effects of the analytic approximations used for the integral $J(X, Y, Z)$ in equation (7.9), and for the integrals performed to obtain equations (7.29) and (7.40), we have evaluated contributions to the decay widths by integrating numerically over the Feynman parameters and the KK mass-squared $m_{\vec{n}}^{2}$, using Mathematica. We find a deviation of approximately $5 \%$ between the analytically approximated and the numerically approximated answers for the decays to spin- 2 excitations, and a deviation of less than $1 \%$

\footnotetext{
${ }^{3}$ Aside from the analytically approximated expressions for the $\tilde{\phi}$ widths, which are calculated by hand, these are evaluated using Mathematica [27]. The infinite sums are evaluated by truncation once the quoted level of significance has been achieved. The numerical approximations to the integrals are evaluated using the NIntegrate function with default options, and (for comparison) using a number of other numerical integration routines [28] with their default options.
} 


\begin{tabular}{|l|l|l|l|l|}
\hline \multirow{2}{*}{$n$} & \multirow{2}{*}{ an. approx. } & \multicolumn{3}{|c|}{$\tilde{h}$ numerical approximation } \\
\cline { 3 - 5 } & full & $m_{f} \rightarrow 0$ & W,t approx. \\
\hline 2 & 0.16 & 0.16 & 0.16 & 0.16 \\
3 & 0.056 & 0.058 & 0.057 & 0.056 \\
4 & 0.019 & 0.020 & 0.020 & 0.019 \\
5 & 0.0066 & 0.0070 & 0.0069 & 0.0066 \\
6 & 0.0020 & 0.0023 & 0.0023 & 0.0022 \\
\hline
\end{tabular}

Table 1: Numerical values of the $\tilde{h}$ contributions inside the square brackets of equation (8.21) for some different values of $n$ ('an. approx.' column), and the equivalent values when the contributions are calculated by numerical integration. The 'full' column shows the results of integrating numerically the full expression for the decay width (without making the approximations made in the analytic case), and is the value with which the analytic expression is compared. The extra columns show the effect of performing numerical integration under the extra approximations of neglecting the masses of the fermions other than the top (' $m_{f} \rightarrow 0$ ' column) and of assuming in addition to this that the $\mathrm{W}$ and top masses dominate the denominators of their respective integrands ('W,t approx.' column). The purpose of including such columns is to give an indication of how much these approximations change the final answer.

\begin{tabular}{|l|l|l|l|l|l|}
\hline \multirow{2}{*}{$n$} & $\tilde{\phi}$ an. approx. & \multicolumn{4}{|c|}{$\tilde{\phi}$ numerical approximation } \\
\cline { 3 - 6 } & 5.3 & 5.3 & 5.3 & 4.4 & 5.4 \\
3 & 3.9 & 4.0 & 3.9 & 3.3 & 4.0 \\
4 & 2.2 & 2.2 & 2.2 & 1.8 & 2.2 \\
5 & 1.0 & 1.0 & 1.0 & 0.85 & 1.1 \\
6 & 0.46 & 0.46 & 0.46 & 0.37 & 0.47 \\
\hline
\end{tabular}

Table 2: Numerical values of the $\tilde{\phi}$ contributions inside the square brackets of equation (8.21) for some different values of $n$ ('an. approx.' column), and the equivalent values when the contributions are calculated by numerical integration. Again the 'full' column gives the values with which those calculated analytically are compared. The extra columns show the effects of neglecting the masses of the fermions other than the top (' $m_{f} \rightarrow 0$ ' column), of assuming in addition to this that the $\mathrm{W}$ and top masses dominate the denominators of their respective integrands ('W,t approx.' column), and of making this assumption but changing the numerators to eliminate $M_{W}^{2}$ as done in the analytic calculation ('Num. change' column; as noted in the text this is equivalent to considering higher order corrections in the series expansions for the fractions in the integrand).

for the decays to spin-0 excitations ${ }^{4}$. We have also calculated numerical approximations subject to the assumptions of zero mass for fermions other than the top, and (additionally

\footnotetext{
${ }^{4}$ The quoted deviations compare the values obtained from analytic approximation with the values obtained from evaluating the full integral numerically using Mathematica's NIntegrate routine.
} 
to the previous assumption) of the $\mathrm{W}$ and top masses dominating denominators in their respective integrands. This indicates that the zero fermion mass assumptions are a very good approximation, but that assuming that the $\mathrm{W}$ and top masses are much greater than half the $\mathrm{Z}$ mass yields a poorer, although still acceptable, approximation. It is also

notable that the numerical approximations of the integrals for the decay into $\tilde{\phi}$ suggest that the accuracy of the analytic calculation of the width is much improved by rewriting the numerators of the approximated fractions as we have.

\section{Conclusions}

We have evaluated amplitudes for Z-photon-Kaluza Klein graviton/gravi-scalar interaction, relevant to extra dimensional models in which the Standard Model fields are confined to a 4-brane.

In addition, we have evaluated in the ADD scenario a reasonable approximation to lowest order for the decay width of a $\mathrm{Z}$ boson to a photon and any Kaluza-Klein excitation of the graviton. This width gives an extra contribution to the channel of $\mathrm{Z}$ boson decay to photon plus missing energy compared with the Standard Model. The channel can provide stronger bounds on the compactification radius $R / 2 \pi$ for small numbers $n$ of extra dimensions.

The consideration of the decay channel involving spin-0 KK excitations (gravi-scalars) has proved significant: for the decays considered, these channels provide the larger contribution to the overall width. The consideration of processes involving the gravi-scalar has not been common in the literature.

We expect our signal to be most significant when the centre of mass energy is equal to $M_{Z}$, as, for example, was the case at LEP. We therefore intend to calculate bounds upon the compactification radius from LEP data [12], given that the data appear to be in accordance with Standard Model predictions. Our process would be additional to the tree-level radiation of a spin-2 KK graviton tower from a photon.

The amplitudes obtained for $Z \rightarrow \tilde{h} \gamma$ and $Z \rightarrow \tilde{\phi} \gamma$ could also be used in the calculation of decays of a RS1 KK mode into a Z boson and a photon. In RS1, the KK mode coupling is enhanced by a warp factor, making resonant production at colliders and interesting signature, worthy of study. Although $\tilde{h} \rightarrow \gamma Z$ and $\tilde{\phi} \rightarrow \gamma Z$ are loop-induced processes and are therefore suppressed with respect to other, tree-level decays of a RS1 KK mode, the channel would be useful for checking the couplings $\kappa$ of the excitations, à la reference [29].

\section{Acknowledgments}

We should like to thank the members of the Cambridge Supersymmetry Working Group for their comments. KS would like to thank the Institute for Particle Physics Phenomenology at Durham University for hospitality offered whilst this paper was in preparation. BCA and JPS are funded by the United Kingdom's Science and Technology Facilities Council. 


\section{A. Dimensional regularization integral identities}

We write $D=4-\epsilon$, and in each case take the limit as $\epsilon \rightarrow 0 . \gamma$ is the Euler-Mascheroni

constant. The integrals on the left are in Minkowski space. The methodology used may be found, for example, in Appendix A.4 of reference [30].

$$
\begin{aligned}
& \mu^{(4-D)} \int \frac{d^{D} l}{(2 \pi)^{D}} \frac{l^{2}}{\left(l^{2}-X^{2}\right)^{3}}=\frac{i}{(4 \pi)^{2}}\left[\frac{2}{\epsilon}-\gamma+\log (4 \pi)-\log \left(\frac{X^{2}}{\mu^{2}}\right)-\frac{1}{2}\right] \\
& \mu^{(4-D)} \int \frac{d^{D} l}{(2 \pi)^{D}} \frac{l^{2} / D}{\left(l^{2}-X^{2}\right)^{3}}=\frac{1}{4} \frac{i}{(4 \pi)^{2}}\left[\frac{2}{\epsilon}-\gamma+\log (4 \pi)-\log \left(\frac{X^{2}}{\mu^{2}}\right)\right] \\
& \mu^{(4-D)} \int \frac{d^{D} l}{(2 \pi)^{D}} \frac{\left(l^{2}\right)^{2}}{\left(l^{2}-X^{2}\right)^{3}}=3 X^{2} \frac{i}{(4 \pi)^{2}}\left[\frac{2}{\epsilon}-\gamma+\log (4 \pi)-\log \left(\frac{X^{2}}{\mu^{2}}\right)+\frac{1}{6}\right] \\
& \mu^{(4-D)} \int \frac{d^{D} l}{(2 \pi)^{D}} \frac{\left(l^{2}\right)^{2} / D}{\left(l^{2}-X^{2}\right)^{3}}=\frac{3 X^{2}}{4} \frac{i}{(4 \pi)^{2}}\left[\frac{2}{\epsilon}-\gamma+\log (4 \pi)-\log \left(\frac{X^{2}}{\mu^{2}}\right)+\frac{2}{3}\right] \\
& \mu^{(4-D) \int \frac{d^{D} l}{(2 \pi)^{D}} \frac{\left(l^{2}\right)^{2} /(D(D+2))}{\left(l^{2}-X^{2}\right)^{3}}}=\frac{X^{2}}{8} \frac{i}{(4 \pi)^{2}}\left[\frac{2}{\epsilon}-\gamma+\log (4 \pi)-\log \left(\frac{X^{2}}{\mu^{2}}\right)+1\right]
\end{aligned}
$$

\section{References}

[1] J.F. Nieves and P.B. Pal, Gravitational decay of the Z-boson, Phys. Rev. D 72 (2005) 093006 hep-ph/0509321.

[2] N. Arkani-Hamed, S. Dimopoulos and G. Dvali, The hierarchy problem and new dimensions at a millimeter, Phys. Lett. B 429 (1998) 263 hep-ph/9803315.

[3] I. Antoniadis, A possible new dimension at a few TeV, Phys. Lett. B 246 (1990) 377.

[4] I. Antoniadis and K. Benakli, Limits on extra dimensions in orbifold compactifications of superstrings, Phys. Lett. B 326 (1994) 69 hep-th/9310151.

[5] I. Antoniadis, K. Benakli and M. Quiros, Production of Kaluza-Klein states at future colliders, Phys. Lett. B 331 (1994) 313 hep-ph/9403290.

[6] G.F. Giudice, R. Rattazzi and J.D. Wells, Quantum gravity and extra dimensions at high-energy colliders, Nucl. Phys. B 544 (1999) 3 hep-ph/9811291.

[7] T. Han, J.D. Lykken and R-J. Zhang, Kaluza-Klein states from large extra dimensions, Phys. Rev. D 59 (1999) 105006 hep-ph/9811350.

[8] E. Ma and J. Okada, How many neutrinos?, Phys. Rev. Lett. 41 (1978) 287 [erratum ibid. 41 (1978) 1759].

[9] K.J.F. Gaemers, R. Gastmand and F.M. Renard, Neutrino counting in $e^{+} e^{-}$collisions, Phys. Rev. D 19 (1979) 1605.

[10] G. Barbiellini, B. Richter and J.L. Siegrist, Radiative $Z^{0}$ production: a method for neutrino counting in $e^{+} e^{-}$collisions, Phys. Lett. B 106 (1981) 414. 
[11] A. Jachołkowska, J. Kalinowski and Z. Wąs, Higher-order QED corrections to $e^{+} e^{-} \rightarrow \nu \bar{\nu} \gamma$ at LEP2, Eur. Phys. J. C 6 (1999) 485 hep-ph/9803375.

[12] B.C. Allanach and J.P. Skittrall, $Z$ boson decay to photon plus Kaluza-Klein graviton: large extra dimensional bounds, arXiv:0709.2929.

[13] M. Acciarri et al. (L3 Collaboration), Search for new physics in energetic single photon production in $e^{+} e^{-}$annihilation at the $Z$ resonance, Phys. Lett. B 412 (1997) 201.

[14] L. Randall and R. Sundrum, A large mass hierarchy from a small extra dimension, Phys. Rev. Lett. 83 (1999) 3370 hep-ph/9905221.

[15] C.D. Hoyle, D.J. Kapner, B.R. Heckel, E.G. Adelberger, J.H. Gundlach, U. Schmidt and H.E. Swanson, Sub-millimeter tests of the gravitational inverse-square law, Phys. Rev. D 70 (2004) 042004 hep-ph/0405262.

[16] E.G. Adelberger (Eöt-Wash group), Sub-millimeter tests of the gravitational inverse square law, hep-ex/0202008.

[17] ALEPH, DELPHI, L3, OPAL Collaborations and LEP Exotica Working Group, Combination of LEP results on direct searches for large extra dimensions, LEP Exotica WG 2004-03, ALEPH 2004-007 PHYSICS 2004-006, DELPHI 2004-033 CONF 708, L3 Note 2798, OPAL Technical Note TN7443.

[18] A. Abulencia et al. (CDF collaboration), Search for large extra dimensions in the production of jets and missing transverse energy in p $\bar{p}$ collisions at $\sqrt{s}=1.96$ TeV, Phys. Rev. Lett. 97 (2006) 171802 hep-ex/0605101.

[19] V.M. Abazov et al. (DØ collaboration), Search for large extra dimensions in the monojet $+\mathbb{E}_{T}$ channel with the DØ detector, Phys. Rev. Lett. 90 (2003) 251802 hep-ex/0302014.

[20] E.A. Mirabelli, M. Perelstein and M.E. Peskin, Collider signatures of new large space dimensions, Phys. Rev. Lett. 82 (1999) 2236 hep-ph/9811337.

[21] J.F. Nieves and P.B. Pal, Gravitational coupling of neutrinos in a medium, Phys. Rev. D 58 (1998) 096005 hep-ph/9805291.

[22] M.J.G. Veltman, Quantum theory of gravitation, in Les Houches 1975: Methods in field theory, North-Holland Publishing 1976.

[23] K. Aoki, Z. Hioki, R. Kawabe, M. Konuma and T. Muta, Electroweak theory - framework of on-shell renormalization and study of higher-order effects, Prog. Theor. Phys. Suppl. 73 (1982) 1 .

[24] J.A.M. Vermaseren, New features of FORM, math-ph/0010025.

[25] W-M. Yao et al. (Particle Data Group), Review of Particle Physics, J. Phys. G 33 (2006) 1.

[26] S. Weinberg, The quantum theory of fields. Volume I: foundations, Cambridge University Press 1995.

[27] S. Wolfram, The Mathematica book, 5th ed. Wolfram Media Press 2003.

[28] T. Hahn, CUBA: a library for multidimensional numerical integration, Comput. Phys. Commun. 168 (2005) 78 hep-ph/0404043.

[29] B.C. Allanach, K. Odagiri, M.J. Palmer, M.A. Parker, A. Sabetfakhri and B.R. Webber, Exploring small extra dimensions at the Large Hadron Collider, J. High Energy Phys. 12 (2002) 039 hep-ph/0211205. 
[30] M.E. Peskin and D.V. Schroeder, An introduction to quantum field theory, Westview Press 1995. 